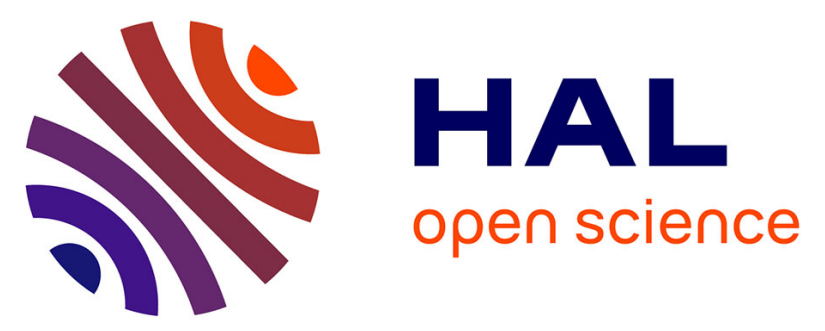

\title{
Lattice-Boltzmann-based large-eddy simulation of high-rise building aerodynamics with inlet turbulence reconstruction
}

Elisa Buffa, Jérôme Jacob, Pierre Sagaut

\section{- To cite this version:}

Elisa Buffa, Jérôme Jacob, Pierre Sagaut. Lattice-Boltzmann-based large-eddy simulation of highrise building aerodynamics with inlet turbulence reconstruction. Journal of Wind Engineering and Industrial Aerodynamics, 2021, 212, pp.104560. 10.1016/j.jweia.2021.104560 . hal-03596056

\section{HAL Id: hal-03596056 https://hal.science/hal-03596056}

Submitted on 3 Mar 2022

HAL is a multi-disciplinary open access archive for the deposit and dissemination of scientific research documents, whether they are published or not. The documents may come from teaching and research institutions in France or abroad, or from public or private research centers.
L'archive ouverte pluridisciplinaire $\mathbf{H A L}$, est destinée au dépôt et à la diffusion de documents scientifiques de niveau recherche, publiés ou non, émanant des établissements d'enseignement et de recherche français ou étrangers, des laboratoires publics ou privés.

\section{(이)(\$)}

Distributed under a Creative Commons Attribution - NonCommercial - NoDerivatives| 4.0 


\title{
Lattice-Boltzmann-based large-eddy simulation of high-rise building aerodynamics with inlet turbulence reconstruction
}

\author{
Elisa Buffa, Jérôme Jacob, Pierre Sagaut * \\ Aix Marseille Univ, CNRS, Centrale Marseille, M2P2 UMR 7340, 13451 Marseille, France
}

\section{A R T I C L E I N F O}

\section{Keywords:}

CFD

Large-eddy simulation

Wind loads

Lattice Boltzmann method

Synthetic eddy method

High rise building

\begin{abstract}
A B S T R A C T
A Lattice-Boltzmann-based Large-Eddy Simulation approach for wind load prediction on high-rise building is proposed and validated. An extension of the original incompressible Synthetic Eddy Method to reconstruct inlet turbulence is proposed within the Lattice-Boltzmann framework, including a low-noise frozen density variant. Extensive successful comparisons with experimental data are carried out, for both quantities defined on the building surface and in its wake. A detailed sensitivity analysis of the results with respect to inlet turbulence reconstruction, boundary conditions at the building surface and grid resolution is also provided. An almost unique set of comparisons with experimental data is presented, including mean and rms values, spectra, but also peak values of pressure at the building surface.
\end{abstract}

\section{Introduction}

Computational Fluid Dynamics is a tool of growing importance in the field of Civil Engineering (Blocken, 2014; Tamura, 2008; Tamura et al., 2008; Dagnew and Bitsuamalak, 2013; Thordal et al., 2019). Among the main applications, one must notice the prediction of wind loads on buildings. At present time, the prediction of the mean (i.e. time averaged) aerodynamic field and related forces exerted on a building is most often performed using the Reynolds-Averaged Numerical Simulation approach. But high-fidelity three-dimensional unsteady approaches like Large-Eddy Simulation (Sagaut, 2005; Garnier et al., 2009) has been shown to have several advantages (Blocken, 2015; Blocken et al., 2016). First, it provides very useful informations about flow and wind load unsteadiness: peak values, dominant frequencies, space-time correlations, which are all of great importance to evaluate the dynamic response of a structure to an unsteady forcing. It is worth noting that LES has also been reported to be more accurate than RANS in predicting time-averaged mean values of aerodynamic forces in some cases as shown in Rodi et al. (1997).

Large-eddy simulation has been used to study building aerodynamics by many research groups during the last decades. Some illustrative examples of application of LES to high-rise buildings, which is the scope of the present article, are displayed in Table 1, which summarizes the inflow generation technique and the quantities used for validation of the results (i.e. the comparison with experimental data). The distinction is made here between data defined at the surface of the building (pressure, aerodynamic forces and their moments ...) and the measurements done in the wake of the building. A first observation is that validation of building-surface-defined quantities (e.g. pressure, aerodynamic forces) and wake flow are almost never done at the same time. A second observation is that both the level and completeness of validation differ a lot depending on the authors. A striking fact is that joined buildingdefined/wake validation are very rare. Most papers are restricted to the third level of validation defined in Sagaut and Deck (2009).

Since the pioneering works performed in the 1970s and early 1980s (Castro and Robins, 1977; Corke and Nagib, 1979; Hillier and Cherry, 1981; Taniguchi et al., 1981; Sakamoto and Arie, 1982; Castro and Dianat, 1983; Bearman and Morel, 1983; Sakamoto, 1985; Sakamoto and Arie, 1983), it is known that aerodynamic forces experienced by an immersed body in a turbulent flow are very sensitive to details of the upstream turbulence, since it depends theoretically on 8 non-dimensional parameters (Martinuzzi and Tropea, 1993) involving features of the upstream flow, among which turbulence intensity, turbulence integral scale and turbulence anisotropy (Blackburn and Melbourne, 1996; Saathoff and Melbourne, 1997; Lim et al., 2007; Hearst et al., 2016; Lim et al., 2009). These works follow the earlier seminal works performed in the 1960s dealing with the theory of vortex shedding mechanics in the wake of bluff bodies and the influence of upstream turbulence, see (Gerrard, 1966; Sumer and Fredsoe, 2006). Therefore, a specific attention must be paid to the capability of synthetic turbulence

\footnotetext{
* Corresponding author.

E-mail address: pierre.sagaut@univ-amu.fr (P. Sagaut).
} 
Table 1

Large-Eddy Simulations of wind load prediction on high-rise building. Inflow: method for reconstruction of turbulent fluctuations on the inlet plane (RFG: family of Random Fourier mode Generator; SEM: family of Synthetic Eddy Method; PS: family of Precursor Simulation methods; RM: family of Recycling/ extraction/rescaling methods; VM: family of Vortex Method). Validation: quantities on which comparisons with reference data are displayed. Validation is related to quantities on which comparisons with reference data are displayed. Building: at the building surface (prime superscript is related to rms quantities, $S$ is related to sprectrum, $C_{p, c o r r}$ denotes two-point spatial correlation of surface pressure); Wake: in the fluid domain in the wake of the building ( $U, V, W$ and TKE refer to mean velocity components and turbulent kinetic energy, respectively).

\begin{tabular}{|c|c|c|c|}
\hline \multirow[t]{2}{*}{ Ref. } & \multirow[t]{2}{*}{ Inflow } & \multicolumn{2}{|l|}{ Validation } \\
\hline & & Building & Wake \\
\hline Huang et al. (2010) & RFG & $C_{D}^{\prime}, C_{L}^{\prime}, C_{M}^{\prime}, S_{F_{x}}(\omega), S_{F_{y}}(\omega), S_{M}(\omega)$ & \\
\hline $\begin{array}{l}\text { Aboshosha et al. (2015); } \\
\text { Elshaer et al. (2016) }\end{array}$ & RFG & $C_{p}, C_{p}^{\prime}, S_{F_{x}}(\omega), S_{F_{y}}(\omega), S_{M}(\omega)$ & \\
\hline Daniels et al. (2013) & RFG & $C_{p}, C_{p}^{\prime}$ & \\
\hline Guichard (2019a) & RFG & $C_{p}, S_{p p}(\omega)$ & \\
\hline Alminhana et al. (2018) & SEM & $C_{D}, C_{p}$ & \\
\hline Pavlidis et al. (2010) & SEM & & $\begin{array}{l}U, W \\
u^{\prime} w^{\prime}\end{array}$ \\
\hline \multirow[t]{2}{*}{$\begin{array}{l}\text { Dagnew and } \\
\text { Bitsuamalak (2014) }\end{array}$} & SEM & $C_{p}, C_{p}^{\prime}, C_{D}, C_{L}, C_{M}$ & \\
\hline & $\begin{array}{l}\text { RFG } \\
\text { PS }\end{array}$ & $\begin{array}{l}C_{D}^{\prime}, C_{L}^{\prime}, C_{M}^{\prime} \\
S_{F_{x}}(\omega), S_{F_{y}}(\omega), S_{M}(\omega)\end{array}$ & \\
\hline Yan and Li (2015) & $\begin{array}{l}\text { RFG } \\
\text { VM } \\
\text { RM } \\
\text { PS }\end{array}$ & $\begin{array}{l}C_{p}, C_{p}^{\prime}, C_{D}, C_{L}, C_{D}^{\prime}, C_{L}^{\prime} \\
S_{F_{x}}(\omega), S_{F_{y}}(\omega), S_{M}(\omega)\end{array}$ & \\
\hline Gousseau et al. (2013) & VM & & $\begin{array}{l}U, V, W, \\
T K E\end{array}$ \\
\hline Nozu et al. (2015) & PS & $C_{p}, C_{p}^{\prime}, C_{D}, C_{L}, C_{D}^{\prime}, C_{L}^{\prime}, S_{F_{x}}(\omega)$ & \\
\hline Guichard (2019b) & PS & $\begin{array}{l}C_{p}, C_{p}^{\prime}, C_{D}, C_{L}, C_{M_{x}}, C_{M_{y}}, C_{M_{z}}, C_{D}^{\prime}, C_{L}^{\prime} \\
C_{M_{x}}^{\prime}, C_{M_{y}}^{\prime}, C_{M_{z}}^{\prime}\end{array}$ & \\
\hline Yan and Li (2017) & $\mathrm{RM}$ & $\begin{array}{l}C_{p}, C_{p}^{\prime}, C_{D}, C_{D}^{\prime}, C_{L}^{\prime} \\
S_{F_{x}}(\omega), S_{F_{y}}(\omega)\end{array}$ & \\
\hline Ricci et al. (2018) & RFG & $C_{p, \text { corr }}, C_{p}, C_{p}^{\prime}, C_{D}, C_{L}, C_{D}^{\prime}, C_{L}^{\prime}$ & \\
\hline Wang and Chen (2020) & RFG & $\begin{array}{l}C_{p}, C_{p}^{\prime}, C_{D}, C_{L}, C_{M_{x}}, C_{M_{y}}, C_{M_{z}}, C_{D}^{\prime}, C_{L}^{\prime} \\
C_{M_{x}}^{\prime}, C_{M_{y}}^{\prime}, C_{M_{z}}^{\prime} S_{F_{x}}(\omega), S_{F_{y}}(\omega), \\
S_{M}(\omega)\end{array}$ & \\
\hline
\end{tabular}

models to reproduce these key features of the physical incoming turbulence and to lead to a reliable prediction of aerodynamic field and loads (Lim et al., 2009; Haque et al., 2014; Luo et al., 2018; Tamura, 2008). It is worth noting that the turbulent inlet issue is also important in RANS simulations (An et al., 2013; Longo et al., 2017).

Since LES is a genuinely unsteady simulation technique, adequate boundary conditions must be derived that will provide the simulation with the required amount of physical informations. The case of turbulent

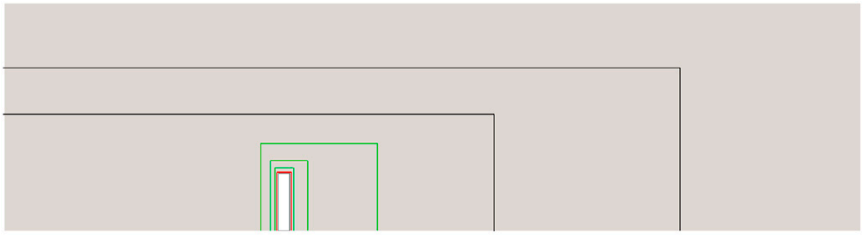

(a) View of the different subdomains used

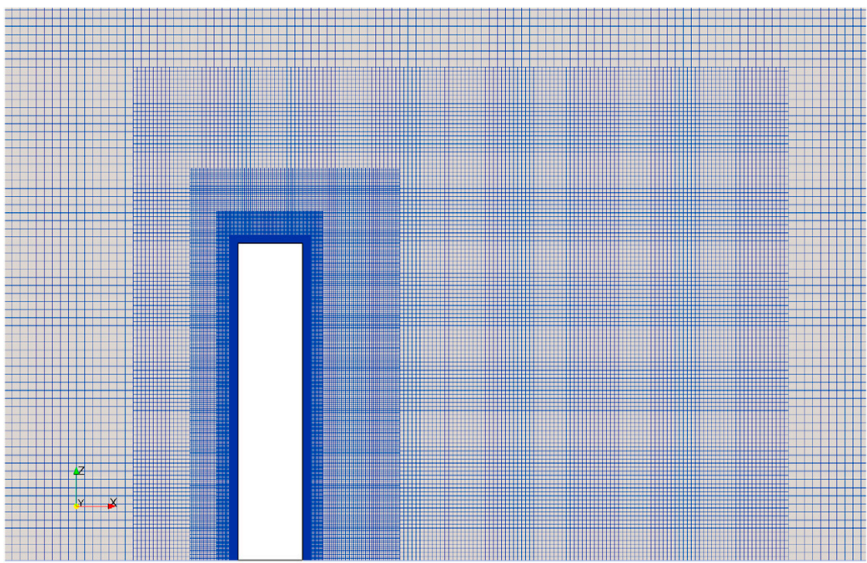

(b) View of the grid around the building

Fig. 2. Visualisation of the different subdomains used in this work with the grid generated in the case of the flow around the building for the fine grid case.

Table 2

Main parameters of numerical setups.

\begin{tabular}{llll}
\hline & Free flow & Tower (coarse grid) & Tower (fine grid) \\
\hline Refinement levels & 3 & 6 & 7 \\
$\Delta x_{\max }^{c} *[-]$ & 0.5 & 0.5 & 0.5 \\
$\Delta x_{\min }^{c}{ }^{*}[-]$ & $1.25 \times 10^{-1}$ & $1.56 \times 10^{-2}$ & $7.81 \times 10^{-3}$ \\
$\Delta t[s]$ & $1.20 \times 10^{-4}$ & $1.50 \times 10^{-5}$ & $7.52 \times 10^{-6}$ \\
Grid points & $2,964,861$ & $5,826,277$ & $14,323,803$ \\
Flow grid points & $2,964,861$ & $5,772,275$ & $14,269,801$ \\
\hline
\end{tabular}

Table 3

SEM parameters considered in order to assess the inlet flow. $U^{c}=8.8 \mathrm{~m} / \mathrm{s}$.

\begin{tabular}{lll}
\hline $\mathrm{N}$ & $\mathrm{L}$ & $\mathrm{K}$ \\
\hline 50 & 0.25 & 200 \\
150 & 0.25 & $10,20,50,100,200$ \\
150 & 0.75 & $1,5,10$ \\
\hline
\end{tabular}

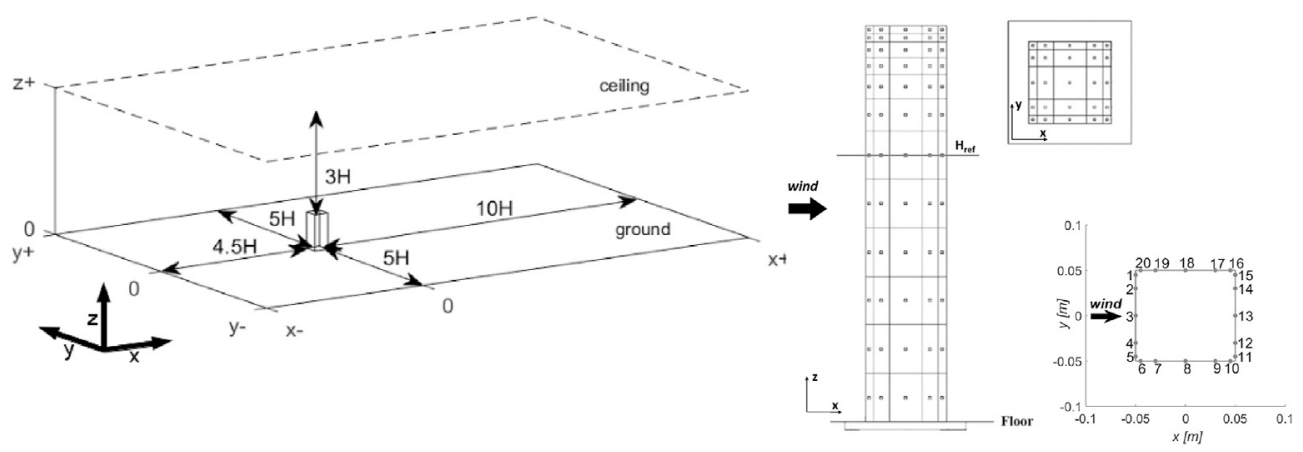

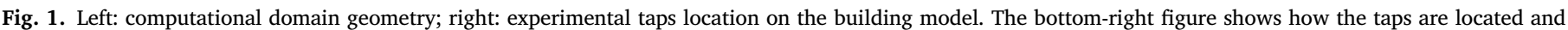
named around the tower's perimeter, considering a plane parallel to the floor. The same nomenclature is kept for the numerical data. 
Table 4

List of the simulations discussed and their main boundary conditions.

\begin{tabular}{|c|c|c|c|c|c|}
\hline \multirow[t]{2}{*}{ Code } & \multicolumn{2}{|c|}{$\begin{array}{l}\text { Turbulence } \\
\text { inlet }\end{array}$} & \multirow[b]{2}{*}{ other } & \multirow[t]{2}{*}{ Wall BC } & \multirow[t]{2}{*}{ Investigated effect } \\
\hline & $\mathbf{L}$ & $\mathbf{K}$ & & & \\
\hline c_20_200_slip & 0.25 & 200 & $\mathrm{x}$ & slip & $\mathrm{L}$ and $\mathrm{K}$ \\
\hline c_75_10_slip & 0.75 & 10 & $\mathrm{x}$ & slip & $\mathrm{L}$ and $\mathrm{K}$ \\
\hline c_0_0_slip & $\mathrm{x}$ & $\mathrm{x}$ & $\mathrm{x}$ & slip & no SEM \\
\hline f_75_10_slip & 0.75 & 10 & $\mathrm{x}$ & slip & Mesh refinement \\
\hline c_75_1_slip & 0.75 & 1 & $\mathrm{x}$ & slip & $\mathrm{K}$ \\
\hline c_75_5_slip & 0.75 & 5 & $\mathrm{x}$ & slip & $\mathrm{K}$ \\
\hline c_75_10_D_slip & 0.75 & 10 & D. d. & slip & $\begin{array}{l}\text { Dirichlet density/ } \\
\mathrm{BC} \text { at wall }\end{array}$ \\
\hline c_75_10_D_noslip & 0.75 & 10 & D. d. & no-slip & $\mathrm{BC}$ at wall \\
\hline c_75_10_D_model & 0.75 & 10 & D. d. & $\begin{array}{l}\text { Wall } \\
\text { model }\end{array}$ & $\mathrm{BC}$ at wall \\
\hline $\begin{array}{l}\text { D.d.: Dirichlet dens } \\
\text { (at inlet) }\end{array}$ & & & & & \\
\hline
\end{tabular}

D.d.: Dirichlet density (at inlet)

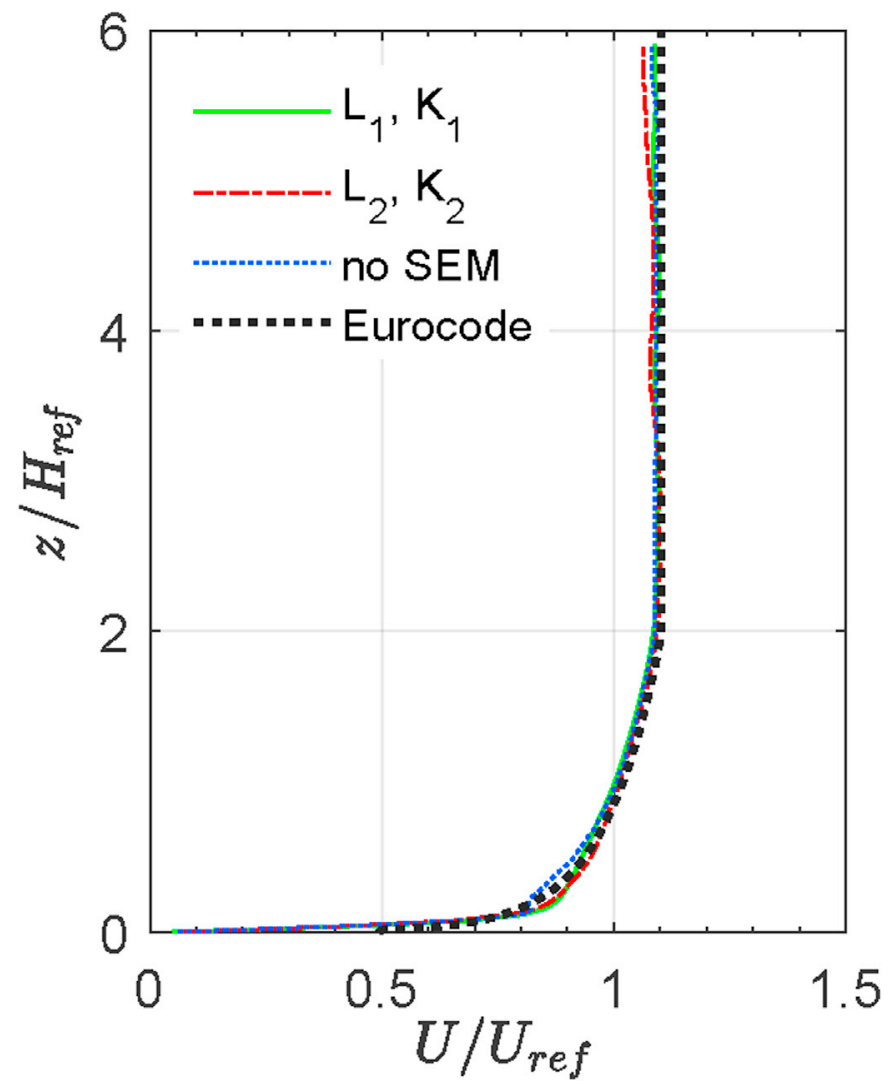

Fig. 3. Mean velocity profile, comparing different inlet conditions.

inflow conditions is known to be an open challenging issue. The key problem is that realistic enough fluctuations must be prescribed at the inlet plane to mimic the incoming physical turbulence. Several approaches for that purpose have been proposed (Sagaut, 2005; Vasaturo et al., 2018; Lamberti et al., 2018; Dhamankar et al., 2018; Luo et al., 2018; Wu, 2016), among which i) the use of precursor simulations, ii) the use of a recycling technique, iii) the use of adaptive forcing terms and iv) the generation of a synthetic fluctuating field. These approaches have been observed to yield satisfactory results in simple configurations, but the most versatile and general one is the last one. Accordingly, the reconstruction of synthetic turbulence is the method selected in the present work, using a Synthetic Eddy Method (Jarrin et al., 2006; Pamiès et al., 2009) in practice.
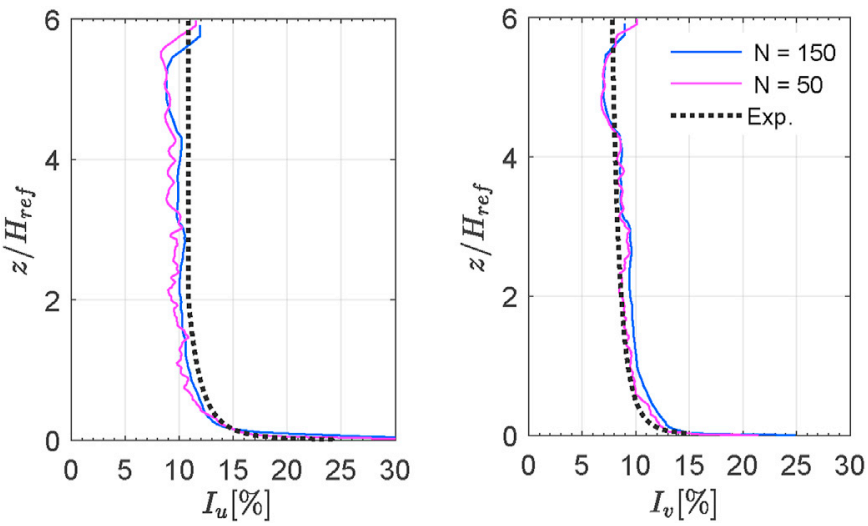

Fig. 4. Effect of $\mathrm{N}$ on the turbulence intensities at the building location $(x, y)=$ $(0,0)$.

The scope of the present paper is twofold. First, the capability of Lattice-Boltzmann-based Large-Eddy Simulation to predict the flow around a high-rise building will be assessed by addressing a configuration on which a huge amount of accurate experimental data is available, and to provide exhaustive comparisons. To this end, the recent wind tunnel experiments of Sheng et al. (2018) are selected. More generally, the purpose of the paper is to provide one among the most exhaustive available cross-comparison of LES and experimental results, giving a deeper insight into the usefulness of LES for wing engineering. Second, the sensitivity of the results to details of the inlet turbulence reconstruction is investigated, giving some insights into the relative importance of the parameters of the method used to generate inlet turbulence and also on the robustness of the present results. To get improved results, a new Reynolds stress rescaling factor is introduced in the SEM, and a new low-noise frozen density version is proposed within the LBM framework.

The paper is organized as follows. First the Synthetic Eddy Methods used for the present work is presented in Section 2. The key features of the Lattice-Boltzmann Method used for large-eddy simulation in the present work are given in Section 3, along with a detailed description of the numerical implementation of SEM and the newly proposed low-noise frozen density version of SEM for LBM and subgrid modelling. Numerical setups are detailed in Section 4. The proposed inflow generation method is first calibrated and assessed by considering the free stream flow without building, at the building location in Section 5. LBM-LES results dealing with both building wake and building surface data are then presented in Section 6, along with a statistical convergence analysis. At last, the sensitivity of the results to the proposed SEM method, the boundary condition used at the building surface and the grid resolution is investigated in Section 7. Conclusions are drawn in Section 8.

\section{Synthetic eddy method for inlet turbulence reconstruction}

The Synthetic Eddy Method (SEM) proposed by Jarrin et al. (2006) is based on the key idea that turbulent fluctuations can be reconstructed by generating a random set of ideal vortices, whose features are tuned in order to recover the targeted distribution of Reynolds stresses and spectra. The use of model vortices instead of random Fourier mode, as done by many authors on the grounds of the seminal works of Kraichnan (1970) and Smirnov et al. (2001) on synthetic turbulence, gives a more physical character to the generated fluctuating field, and shares some features with Vortex Methods in CFD. The initial SEM has been modified and extended in many ways, e.g. to account for the existence of different vortex families in turbulent boundary layers (Pamiès et al., 2009; Roidl et al., 2013) or to account for multiple vortex size (Luo et al., 2017). A recent survey of the variants of SEM is available in Skillen et al. (2016).

The method used here is based on the original SEM of Jarrin et al. 


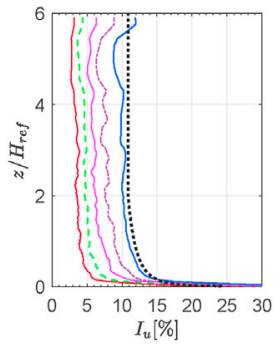

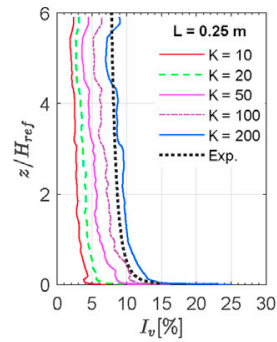

(a)
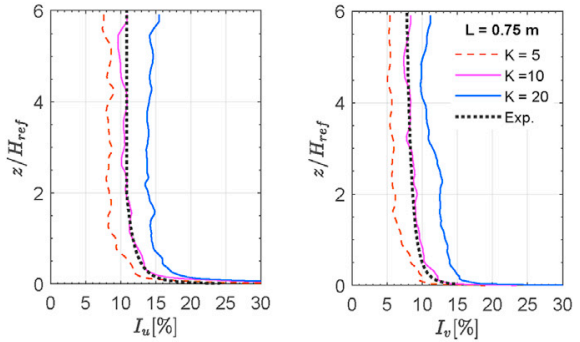

(b)

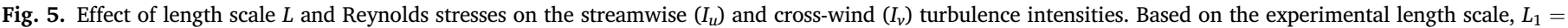
$0.25 \mathrm{~m}$ (a) and $L_{2}=0.75 \mathrm{~m}$ (b) are considered.

(2006), which has already been successfully assessed for the simulation of atmospheric flows for wind engineering purposes (Pavlidis et al., 2010; Creech et al., 2015; Aristodemou et al., 2018; Woodward et al., 2019). In the present paper, a new rescaling factor for the Reynolds stresses is introduced, in order to recover the best possible agreement between the simulated free stream turbulence and experimental data at the location of the building, and not only on the inlet plane.

An isotropic fluctuating field $\tilde{\boldsymbol{u}}(x, y, z, t)$ at location $(x, y, z)$ and time $t$ on the inlet plane is first generated as follows

$\tilde{u}_{j}(x, y, z, t)=\frac{1}{\sqrt{N}} \sum_{i=1, N} \epsilon_{i j} f\left(\tilde{x}_{i}\right) f\left(\tilde{y}_{i}\right) f\left(\tilde{z}_{i}\right)$

with $N$ the number of vortices used to generate the fluctuations, $\epsilon_{i j}$ the sign of vortex $i$ on component $j$ of $\tilde{\boldsymbol{u}}$ and $f$ the vortex shape function. The coordinate $\tilde{x}_{i}, \tilde{y}_{i}, \tilde{z_{i}}$ are then defined following

$\tilde{x}_{i}=\left(x-x_{i}\right) / L$

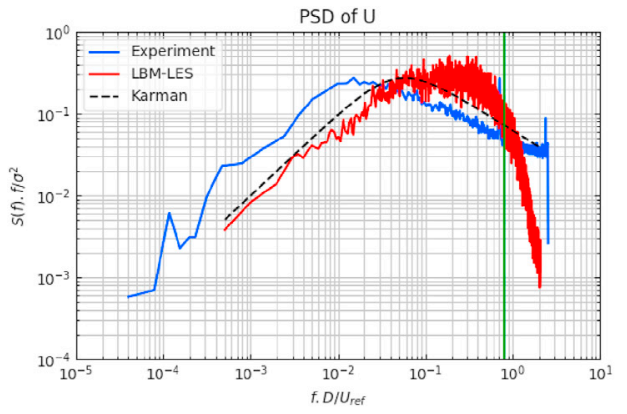

(a)

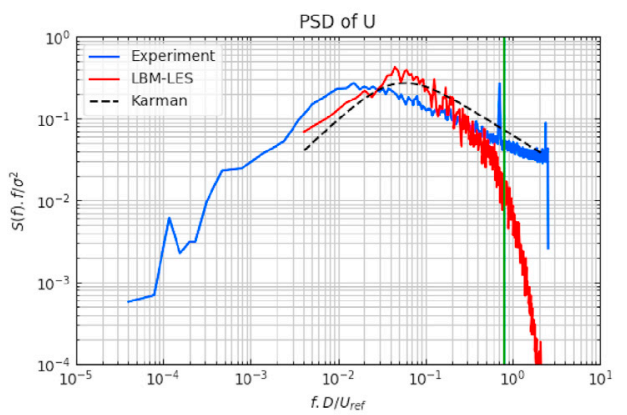

(b)

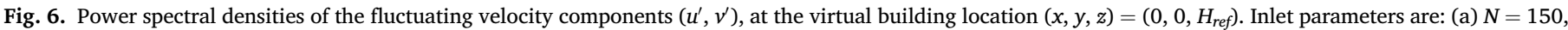

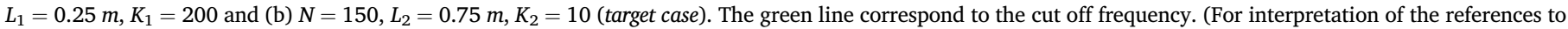
color in this figure legend, the reader is referred to the Web version of this article.)

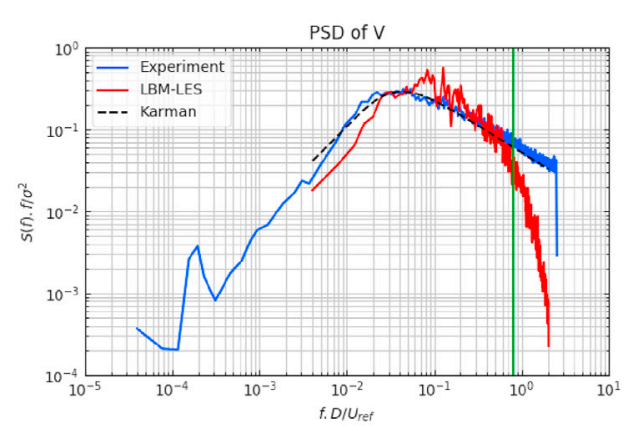

$\tilde{y}_{i}=\left(y-y_{i}\right) / L$

$\tilde{z_{i}}=\left(z-z_{i}\right) / L$

where $L$ denotes the characteristics length of vortex $i$ and $x_{i}, y_{i}, z_{i}$ its coordinate.

The fluctuating velocity field $\boldsymbol{u}^{\prime}(x, y, z, t)$ that corresponds to the Reynolds stress tensor $R_{i, j}(x)$ is then computed as $u_{i}^{\prime}=A_{i j} \tilde{u}_{j}$, where the matrix $\boldsymbol{A}$ is the Cholesky decomposition of the prescribed Reynolds stress tensor:

$\boldsymbol{A}=K\left(\begin{array}{ccc}\sqrt{R_{11}} & 0 & 0 \\ R_{21} / A_{11} & \sqrt{R_{22}-A_{21}^{2}} & 0 \\ R_{31} / A_{11} & \left(R_{32}-A_{21} A_{31}\right) / A_{22} & \sqrt{R_{33}-A_{31}^{2}-A_{32}^{2}}\end{array}\right)$

where $K$ is a new rescaling factor introduced in the present work.

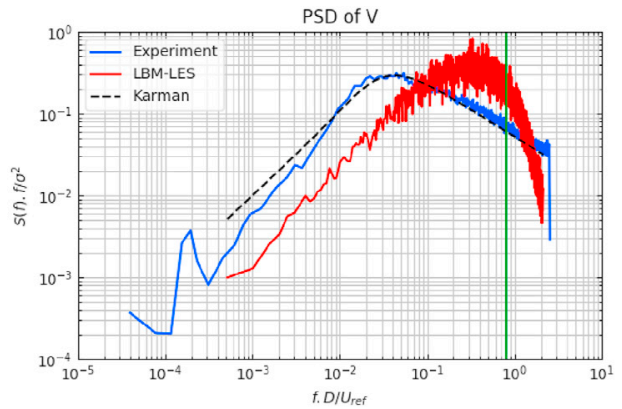



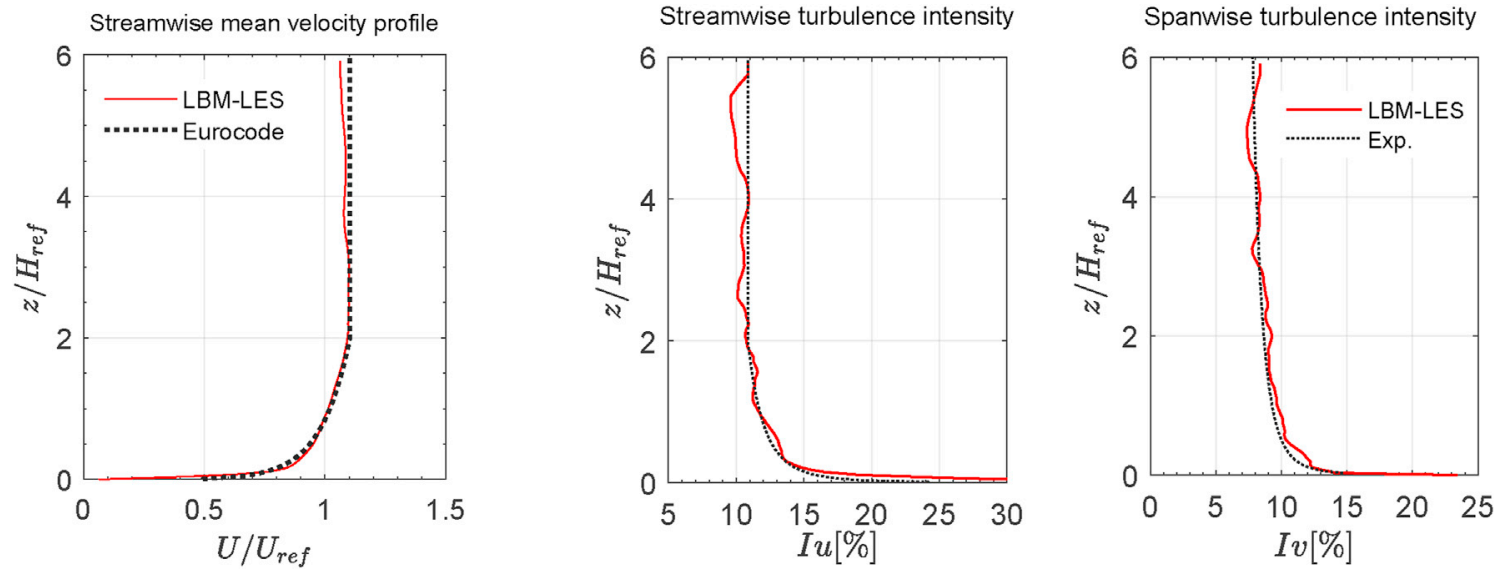

Fig. 7. Turbulent flow statistics at $(x, y)=(0,0)$ : comparison between the experimental measurements and the numerical results of the target case.
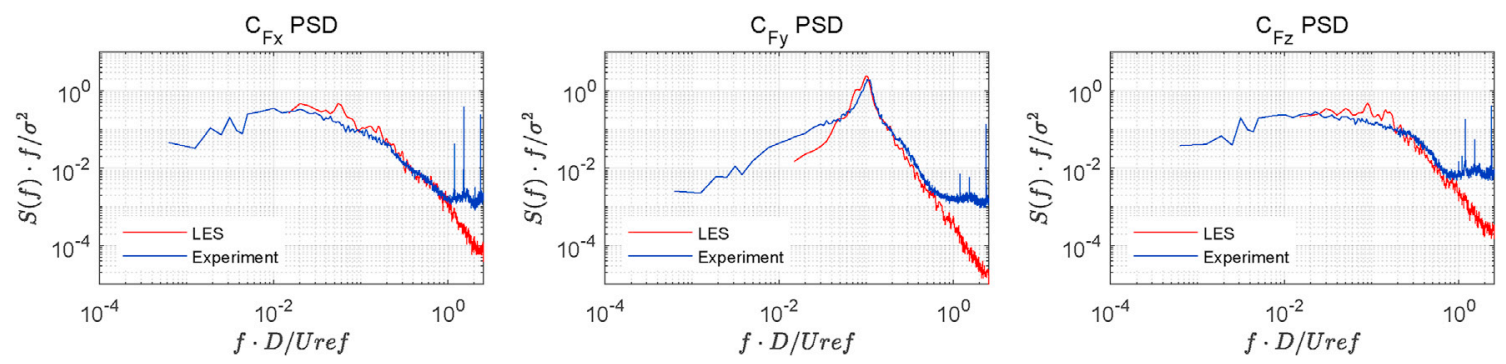

Fig. 8. Power spectral densities for the global forces coefficients (case c_75_10_D_noslip).

An isotropic Gaussian shape function is used, yielding

$f(\tilde{x})=2 \exp \left(\frac{-1}{2 \sigma^{2}} \tilde{x}^{2}\right)$

with $\sigma=0.225$.

The synthetic vortices are assumed to be passively advected in the streamwise direction at a uniform speed $U^{c}$, computed as $0.8 \cdot U_{\infty}=8.8$ $\mathrm{m} / \mathrm{s}$, where $U_{\infty}$ is the freeflow wind speed (Pamiès et al., 2009).

The resulting total velocity field at the inlet is therefore given by

$\boldsymbol{u}(\boldsymbol{x}, t)=\boldsymbol{U}(\boldsymbol{x})+\boldsymbol{u}^{\prime}(\boldsymbol{x}, t)$
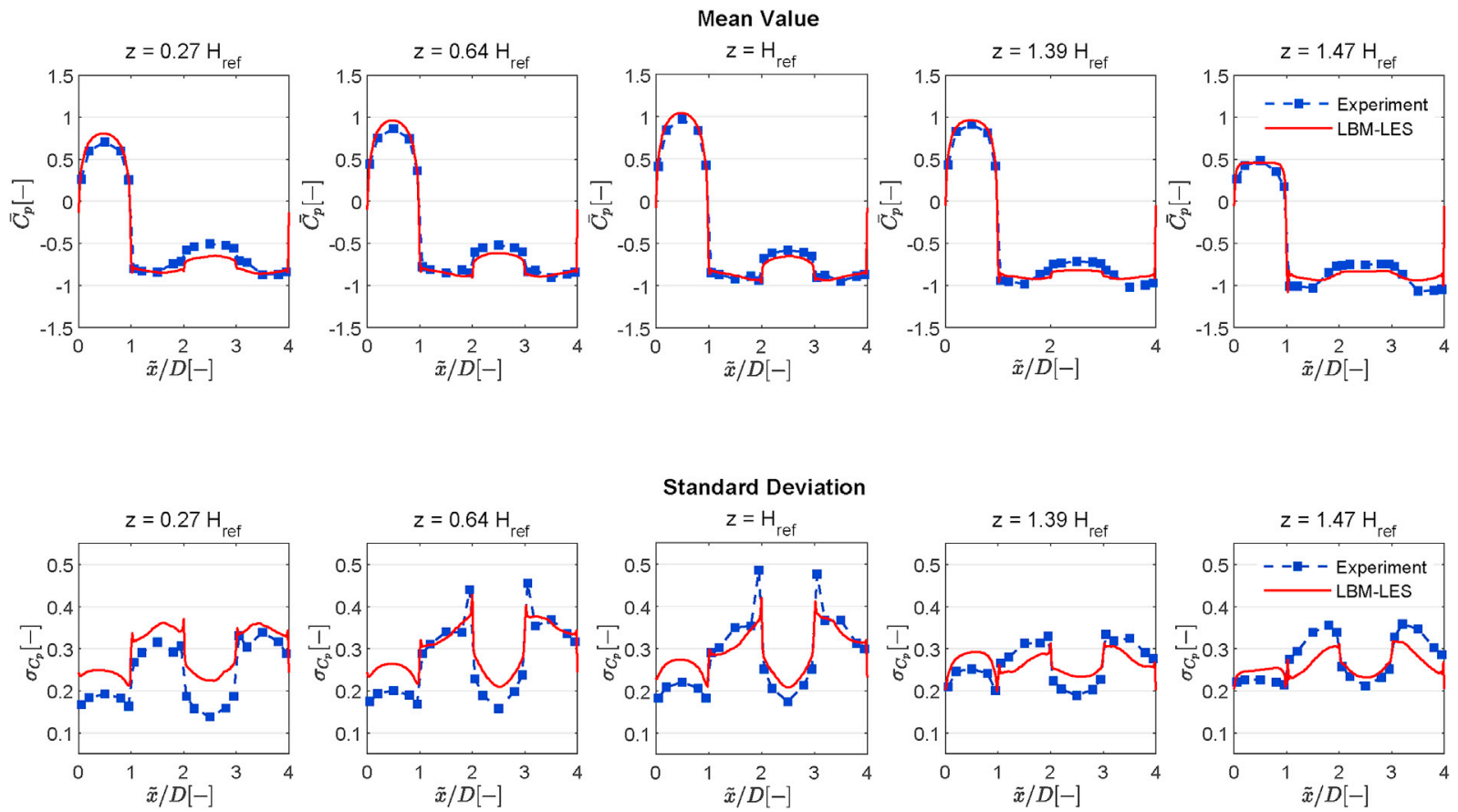

Fig. 9. Mean and rms pressure coefficients around the tower diameters, at five different heights. The heights corresponds to those of the pressure taps in the experimental setup. 


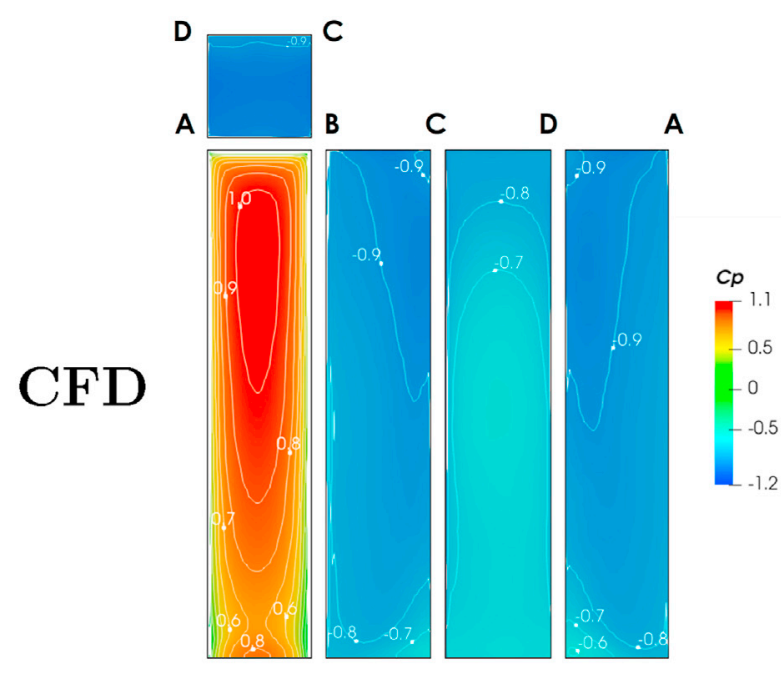

(a)

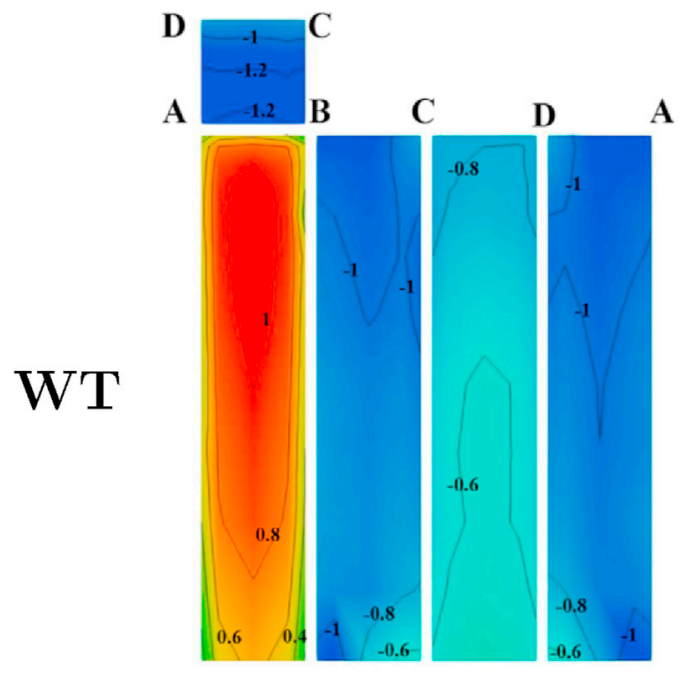

(c)

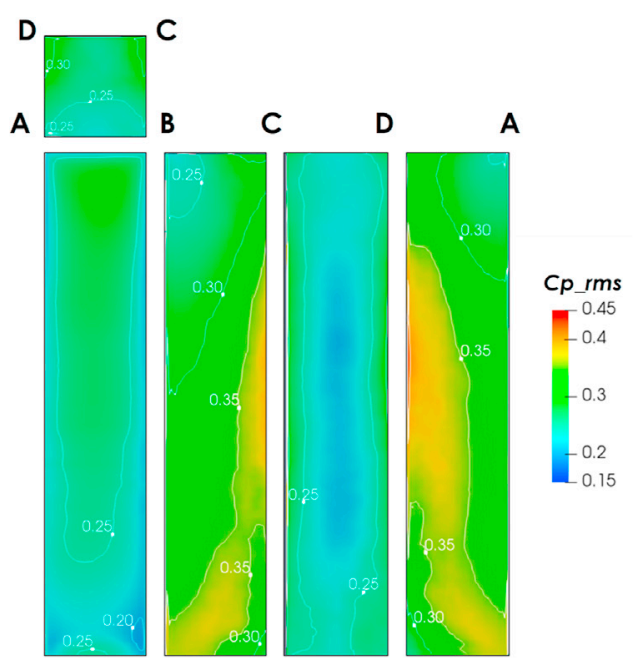

(b)

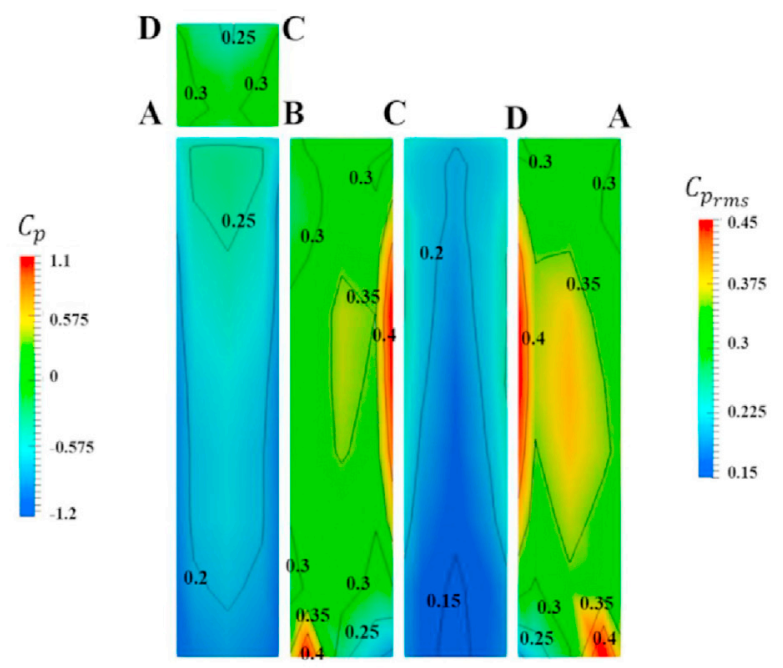

(d)

Fig. 10. Mean (a,c) and standard deviation (b,d) of $C_{p}$ on the tower's sides, comparing the numerical results (a,b) with the experimental ones (c,d). From left to right: front, right, back, left and top facades.

It is worth noting here that the presented synthetic eddy method is based on 3 parameters $(\mathrm{N}, \mathrm{L}$ and $\mathrm{K})$ that may have an impact on the properties of the generated turbulent flow in term of turbulent intensities and spectral contents. Following the procedure described in Yan and Li (2015), numerical simulation will be done first in an empty domain in Section 5 in order to calibrate these different parameters and to analyze their influence on the flow properties, then an obstacle will be added in the domain in Section 6.

\section{Lattice-Boltzmann solver and implementation of SEM}

\subsection{Reminder of the key elements of the Lattice-Boltzmann Method}

Simulations performed in the present article are based on the LatticeBoltzmann method (see Guo and Shu (2013); Krüger et al. (2017) for a comprehensive introduction). Following this mesoscopic approach, a set of coupled partial differential equations for the advection and collision of fluid particles with a finite set of probability density distribution functions $f_{i}$. Macroscopic quantities are reconstructed computing the moments of distribution functions, e.g. $\rho=\sum_{i} f_{i}, \quad \rho \boldsymbol{u}=\sum_{i} c_{i} f_{i}$

where $\boldsymbol{c}_{\boldsymbol{i}}$ is the flow-independent discrete velocity associated to the distribution function $f_{i}$. The general formulation of the governing equation of the Lattice Boltzmann Method is

$\frac{\partial f_{i}}{\partial t}+\boldsymbol{c}_{i} \cdot \nabla f_{i}=C\left(f_{i}\right)$

where $C\left(f_{i}\right)$ is related to the collision term which accounts for the interaction between the fluid particules. In the present case, the Hybrid Recursive Regularized (HRR) model introduced in Jacob et al. (2018) is used for the collision term, since this model exhibits very good stability and accuracy properties, including for strongly convective turbulent flows. This collision model has been extended to account for thermal effects such as compressibility and thermodynamics (Feng et al., 2019b; Guo et al., 2020), in order to obtain a micrometeorology model (Feng et al., 2019a) well suited for the simulation of urban flow physics. In the present work these thermal effects are not taken into account. Therefore, the standard athermal HRR model of Jacob et al. (2018) is used as it was 

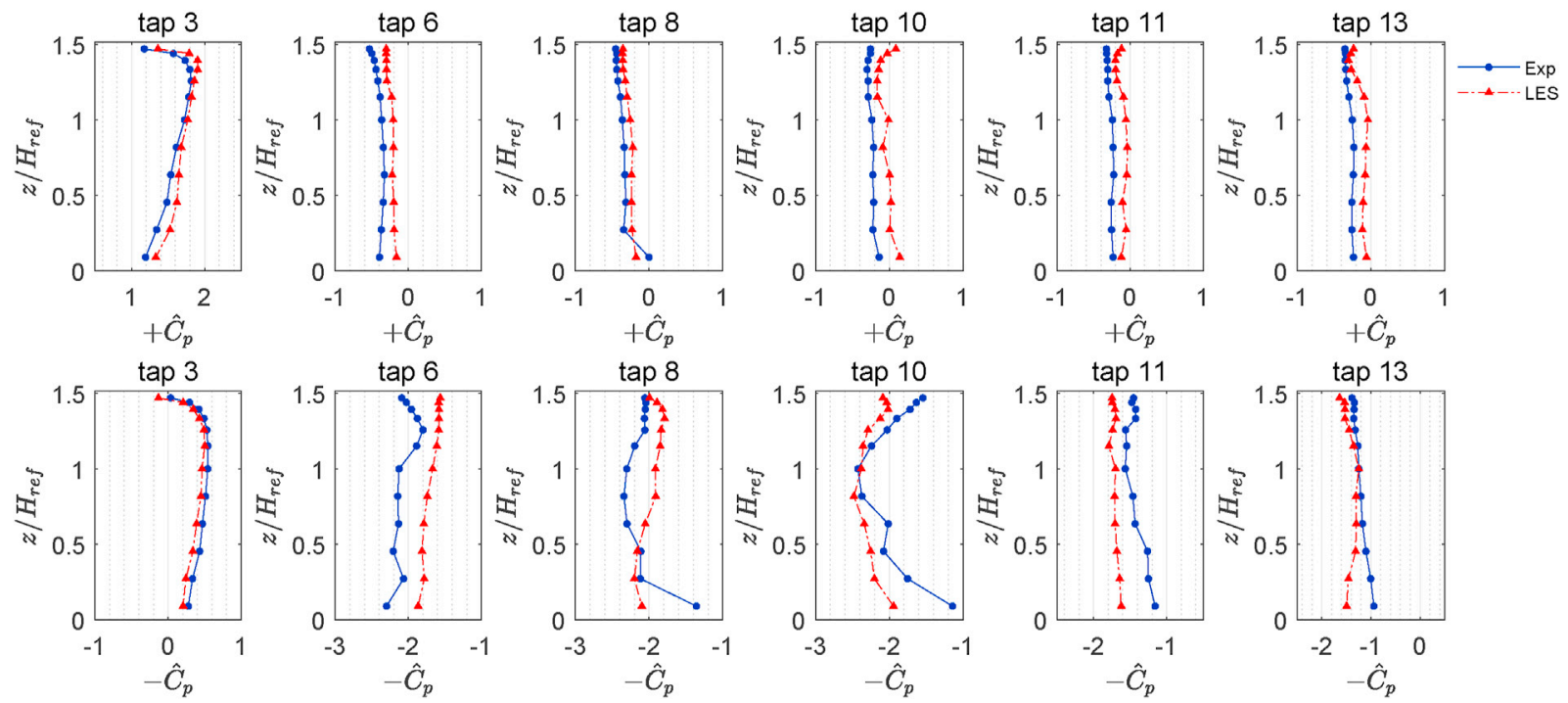

Fig. 11. Local instantaneous wall pressure peak values along the tower's height, considering different locations around the perimeter. The numerical results are compared to the experimental data.
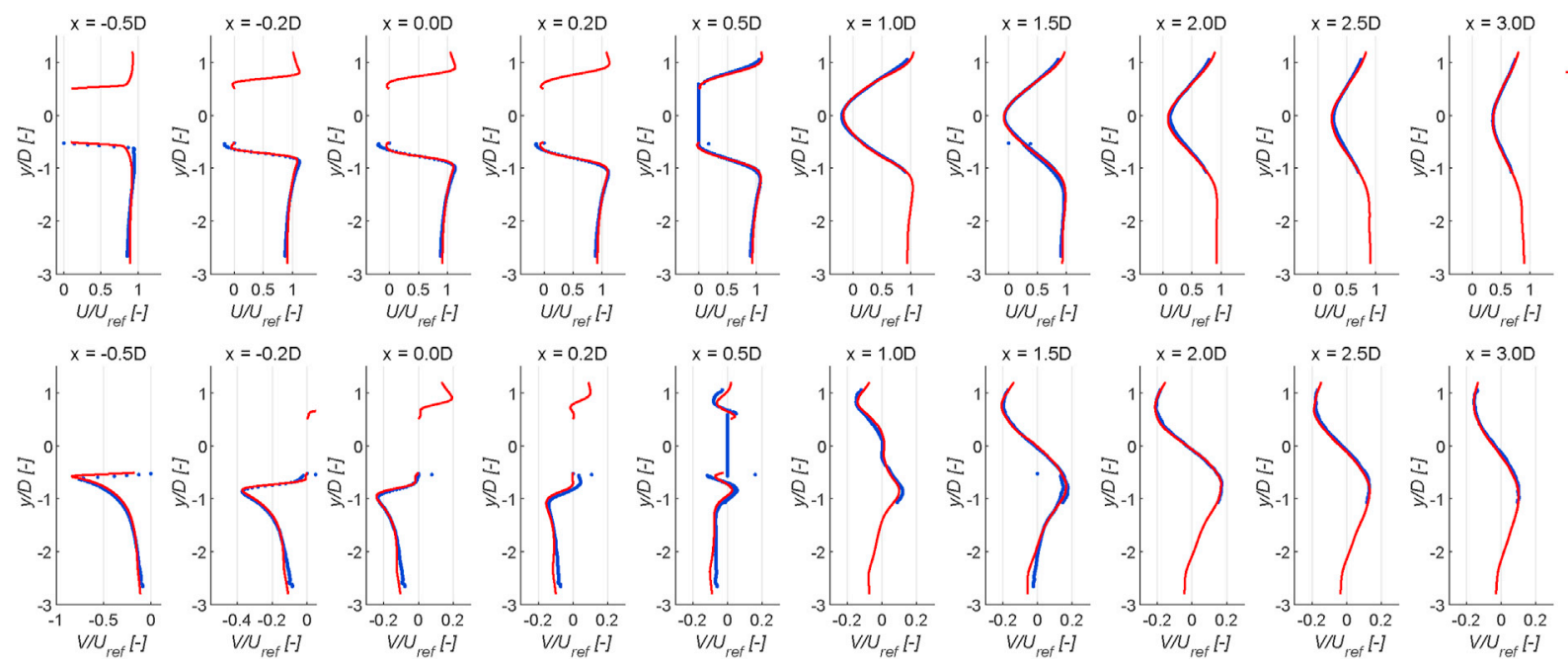

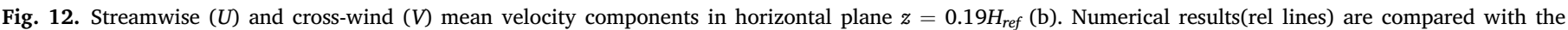
experimental data (blue lines). (For interpretation of the references to color in this figure legend, the reader is referred to the Web version of this article.)

done previously for pedestrian wind comfort study (Jacob and Sagaut, 2018) or atmospheric pollutant dispersion (Merlier et al., 2019). Details of implementation of outlet and wall boundary conditions are available in Feng et al. (2019c), and will not be repeated here for the sake of brevity. Details of the implementation of the SEM are given in the next section.

\subsection{Subgrid modelling and wall modelling for large-eddy simulation}

In the present work, the dynamic version of the HRR collision model given in Jacob et al. (2018) is used to implement the Vreman's subgrid viscosity model for large-eddy simulation (Vreman, 2004), which is defined as

$\nu_{t}=c \sqrt{\frac{B_{\beta}}{\alpha_{i j} \alpha_{i j}}}$,

where $\alpha_{i j}=\frac{\partial u_{i}}{\partial x_{j}}, \quad \beta_{i j}=\Delta^{2} \alpha_{i j} \alpha_{i j}$

and

$B_{\beta}=\beta_{11} \beta_{22}-\beta_{12}^{2}+\beta_{11} \beta_{33}-\beta_{13}^{2}+\beta_{22} \beta_{33}-\beta_{23}^{2}$

The cutoff length $\Delta$ is taken equal to the mesh size, which is particularly relevant for LES-LBM, since LBM relies on the use of cubic cells. The model constant is taken equal to $c=2.5 C_{S}^{2}$, where $C_{S}=0.18$ is the Smagorinsky constant.

Since high-Reynolds LES of wall-bounded flows are addressed, a wall model is used in some simulations presented below. It is based on Afzal's law that account for effects of adverse pressure gradients on turbulent boundary layers, leading to the following velocity profile

$u^{+}=\frac{1}{\kappa} \ln \left(y^{+}\right)+C-\frac{2}{\kappa} \ln \left(\frac{\sqrt{1+p^{+} y^{+}}+1}{2}\right)+\frac{2}{\kappa}\left(\sqrt{1+p^{+} y^{+}}-1\right)$

where the + superscript is related to wall units and the pressure gradient parameter is defined as: 

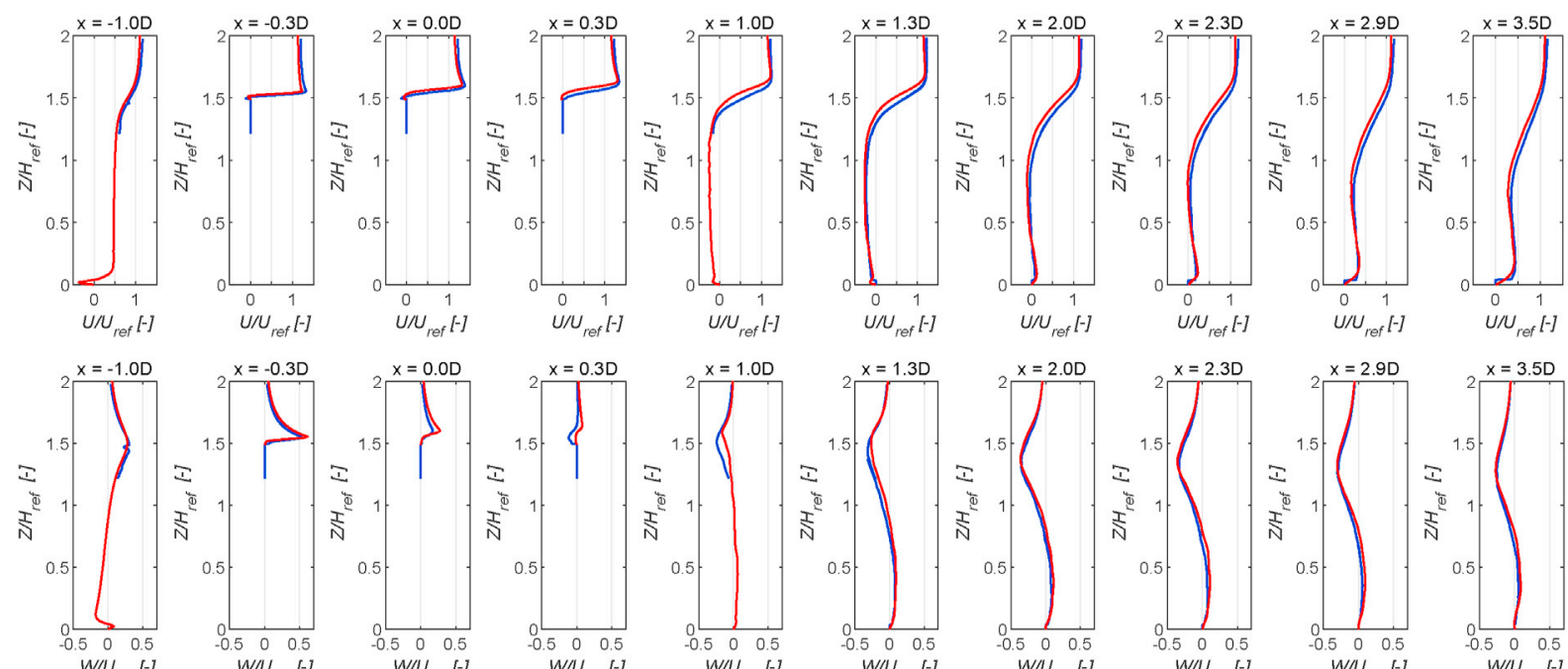

Fig. 13. Streamwise $(U)$ and vertical $(W)$ mean velocity components at the symmetry plane. Numerical results (red lines) are compared with the experimental data (blue lines). (For interpretation of the references to color in this figure legend, the reader is referred to the Web version of this article.)
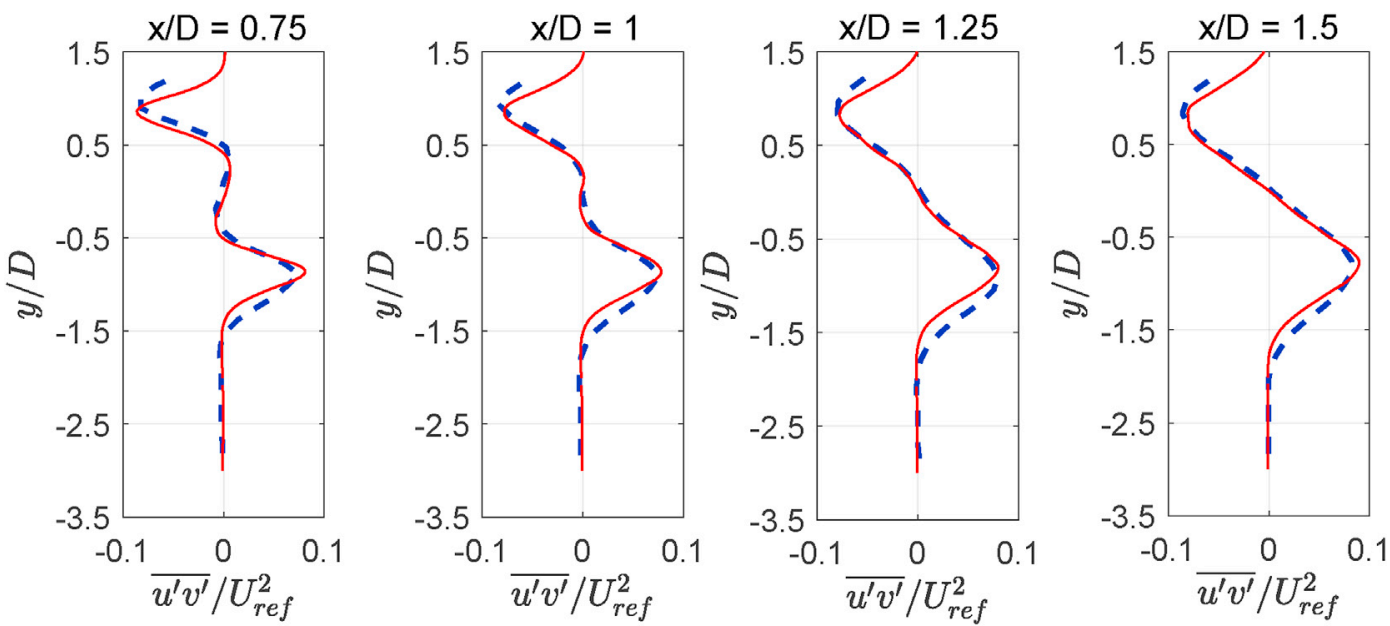

Fig. 14. Turbulent shear stress $u^{\prime} v^{\prime}$ in the wake in the horizontal plane at $z=H_{\text {ref, }}$, for the numerical results and the experimental measurements.

Table 5

Mean values and standard deviations $(\sigma)$ of global force coefficients. c_75_10_D_noslip corresponds the reference simulation discussed above; c_0_0_slip is the case with a steady inflow, i.e. without synthetic turbulence.

\begin{tabular}{|c|c|c|c|c|c|c|}
\hline Case & $\begin{array}{l}\$\{\mid \text { bar }\{\mathrm{C}\}\}_{-}\left\{\mathrm{F}_{-}\right. \\
\{\mathrm{x}\}\}<\text { linline }>\end{array}$ & $\begin{array}{l}\$\{\mid \text { bar }\{\mathrm{C}\}\}_{-} \\
\left\{\{\mid \text {boldsymbol }\{\mathrm{F}\}\}_{-}\right. \\
\{\mathrm{y}\}\}<\text { linline }>\end{array}$ & $\begin{array}{l}\$\{\mid \text { bar }\{\mathrm{C}\}\}_{-} \\
\left\{\{\backslash \text { boldsymbol }\{\mathrm{F}\}\}_{-}\right. \\
\{\mathrm{z}\}\}<\text { linline }>\end{array}$ & $\begin{array}{l}\$\{\mid \text { boldsymbol }\{\backslash \text { sigma }\} \\
\\
-\left\{\mathrm{CF}_{-}\{\mathrm{x}\}\right\}<\text { linline }>\end{array}$ & $\begin{array}{l}\$\{\mid \text { boldsymbol }\{\backslash \text { sigma }\} \\
\left\{\left\{F_{-}\{y\}\right\}<<\text { inline }>\right.\end{array}$ & $\begin{array}{l}\$\{\mid \text { boldsymbol }\{\backslash \text { sigma }\}\} \\
\_\left\{\mathrm{CF}_{-}\{\mathrm{z}\}\right\}<\text { linline }>\end{array}$ \\
\hline c_20_200_slip & 1.042 & 0.013 & 0.191 & 0.226 & 0.305 & 0.067 \\
\hline f_75_10_slip & 1.279 & 0.004 & 0.203 & 0.314 & 0.460 & 0.055 \\
\hline c_75_1_slip & 1.038 & -0.009 & 0.121 & 0.082 & 0.099 & 0.015 \\
\hline c_75_5_slip & 1.135 & -0.003 & 0.159 & 0.185 & 0.264 & 0.032 \\
\hline
\end{tabular}

$p^{+}=\frac{\nu}{\rho u_{\tau}^{3}} \frac{d p}{d s}$

with $u_{\tau}$ the friction velocity at the wall and $d p / d s$ the tangential pressure gradient. The von Karman constant $\kappa$ is taken equal to 0.41 . Practical details of the implementation of the wall law in Lattice-Boltzmann-based Large Eddy simulation are given in Malaspinas and Sagaut (2014) and
Wilhelm et al. (2018) and will not be repeated here for the sake of brevity.

\subsection{Implementation of the synthetic eddy method in the lattice Boltzmann framework}

The implementation of the SEM within the LBM framework is now 
Table 6

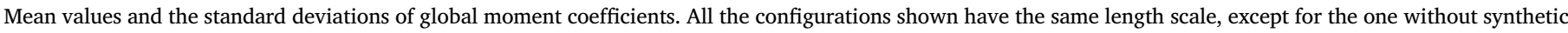
turbulence. As expected, $C_{M_{y}}$ shows the highest values and the increasing of turbulence results into higher standard deviations.

\begin{tabular}{|c|c|c|c|c|c|c|}
\hline Case & $\begin{array}{l}\$\{\mid \operatorname{bar}\{\mathrm{C}\}\}_{-} \\
\left\{\{\backslash \text { boldsymbol }\{\mathrm{M}\}\}_{-}\right. \\
\{\mathrm{x}\}\}<\text { linline }>\end{array}$ & $\begin{array}{l}\$\{\mid \text { bar }\{\mathrm{C}\}\}_{-} \\
\left\{\{\backslash \text { boldsymbol }\{\mathrm{M}\}\}_{-}\right. \\
\{\mathrm{y}\}\}<\text { linline }>\end{array}$ & $\begin{array}{l}\$\{\backslash \text { bar }\{\mathrm{C}\}\}_{-} \\
\left\{\{\mid \text {boldsymbol }\{\mathrm{M}\}\}_{-}\right. \\
\{\mathrm{z}\}\}<\text { linline }>\end{array}$ & $\begin{array}{l}\$\{\mid \text { boldsymbol } \\
\{\backslash \text { sigma }\}\}_{\_}\left\{\mathrm{CM}_{-}\right. \\
\{\mathrm{x}\}\}<\text { inline }>\end{array}$ & $\begin{array}{l}\$\{\backslash \text { boldsymbol }\{\backslash \text { sigma }\}\}_{-} \\
\left\{\{\backslash \text { boldsymbol }\{\mathrm{C}\}\} \mathrm{M}_{-}\right. \\
\{\mathrm{y}\}\}<\text { linline }>\end{array}$ & $\begin{array}{l}\$\{\backslash \text { boldsymbol }\{\backslash \text { sigma }\}\}_{-} \\
\left\{\{\backslash \text { boldsymbol }\{\mathrm{C}\}\} \mathrm{M}_{-}\right. \\
\{\mathrm{z}\}\}<\text { linline }>\end{array}$ \\
\hline c_75_10_slip & -0.015 & 0.605 & -0.003 & 0.179 & 0.115 & 0.026 \\
\hline c_0_0_slip & 0.020 & 0.512 & -0.003 & 0.008 & 0.005 & 0.001 \\
\hline f_75_10_slip & -0.004 & 0.670 & -0.001 & 0.232 & 0.163 & 0.037 \\
\hline c_75_1_slip & 0.024 & 0.546 & -0.003 & 0.053 & 0.045 & 0.012 \\
\hline c_75_5_slip & 0.008 & 0.592 & -0.003 & 0.135 & 0.098 & 0.020 \\
\hline c_75_10_D_slip & -0.006 & 0.611 & -0.003 & 0.186 & 0.113 & 0.025 \\
\hline c_75_10_D_noslip & -0.024 & 0.750 & -0.003 & 0.198 & 0.129 & 0.029 \\
\hline c_75_10_D_model & -0.016 & 0.697 & -0.003 & 0.163 & 0.132 & 0.030 \\
\hline
\end{tabular}
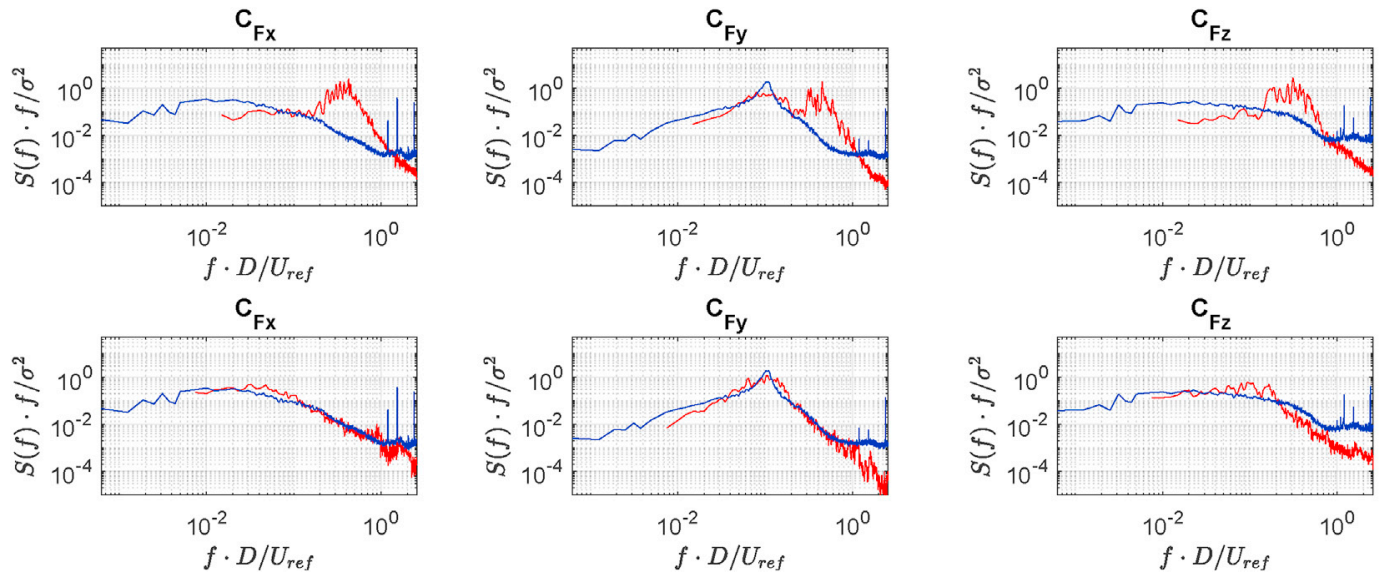

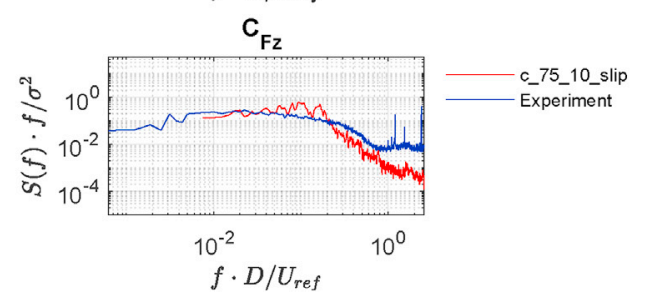

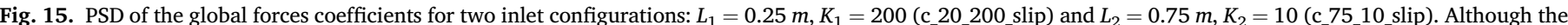
turbulence intensities $\left(I_{u}, I_{v}\right)$ are the same as the experimental ones, but higher Reynolds stresses introduce noise at high frequency.

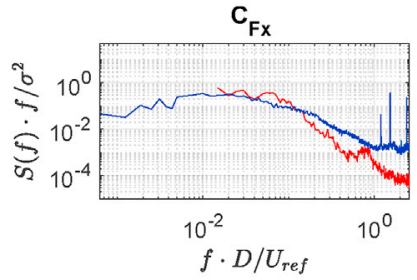

$\mathrm{C}_{\mathrm{Fx}}$

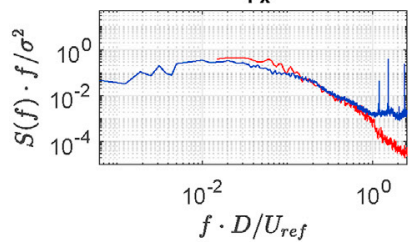

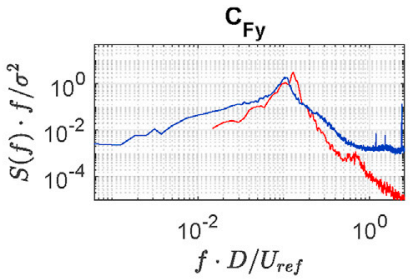

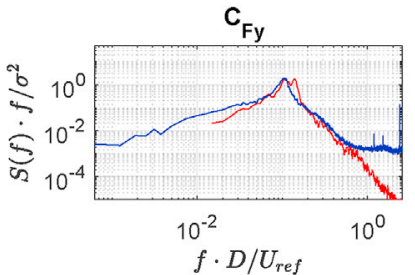

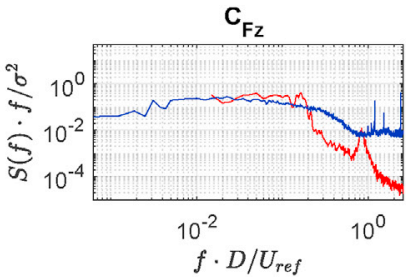

$\mathrm{C}_{\mathrm{Fz}}$

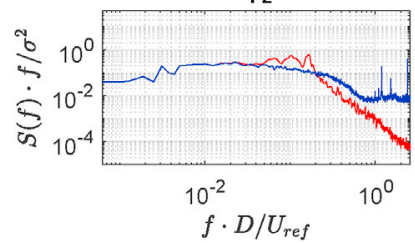

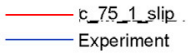

C.75_5_slip Experiment

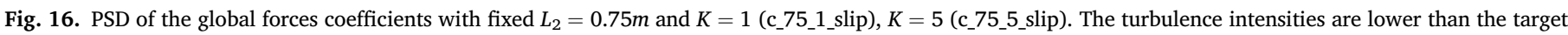
case, and consequently the frequency content is decreased.
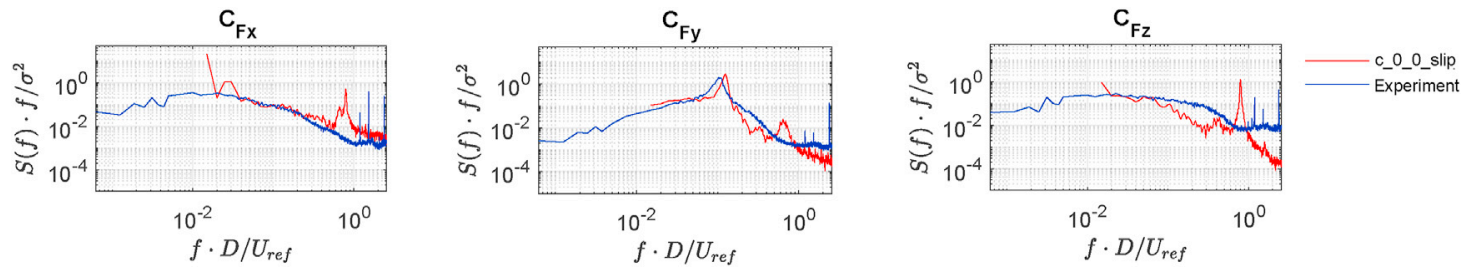

Fig. 17. PSD of the global forces without employing the SEM (c_0_0_slip). 

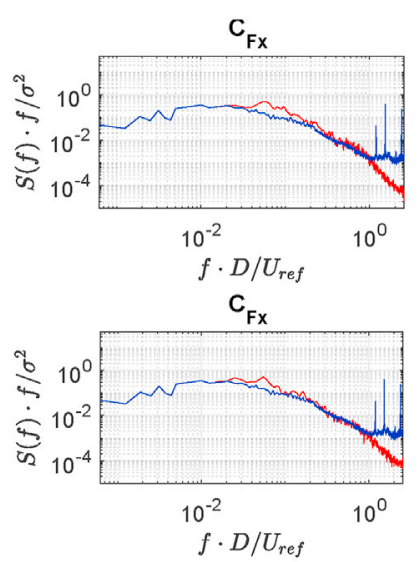

$\mathrm{C}_{\mathrm{FX}}$

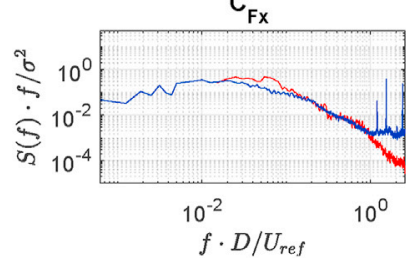

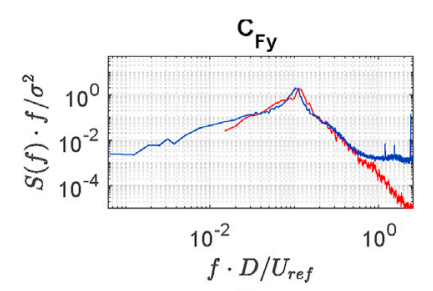
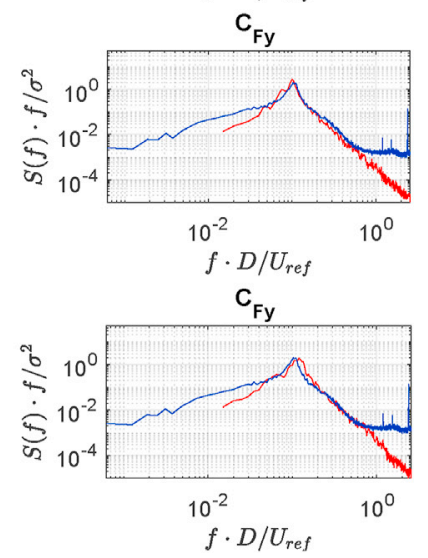
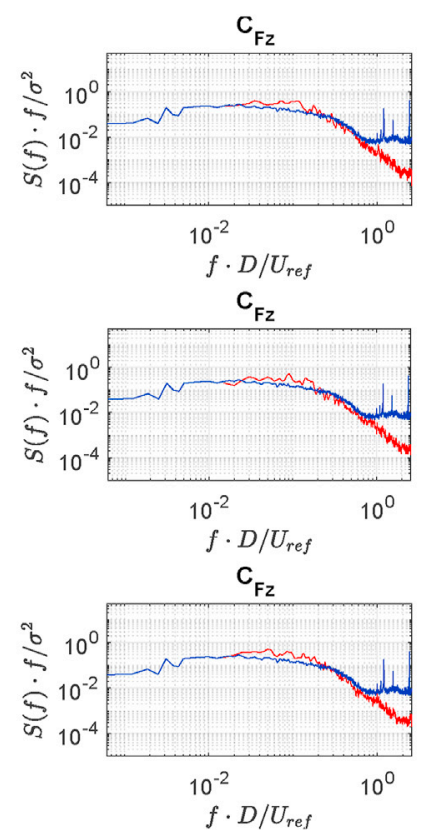
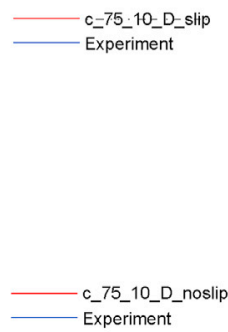

C_ C_75_10_D_model Experiment

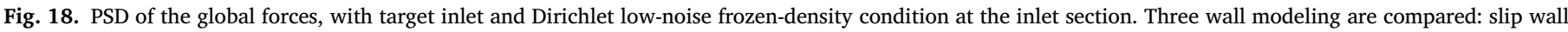
(c_75_10_D_slip), no-slip wall (c_75_10_D_noslip), turbulent Wall model (c_75_10_D_model).
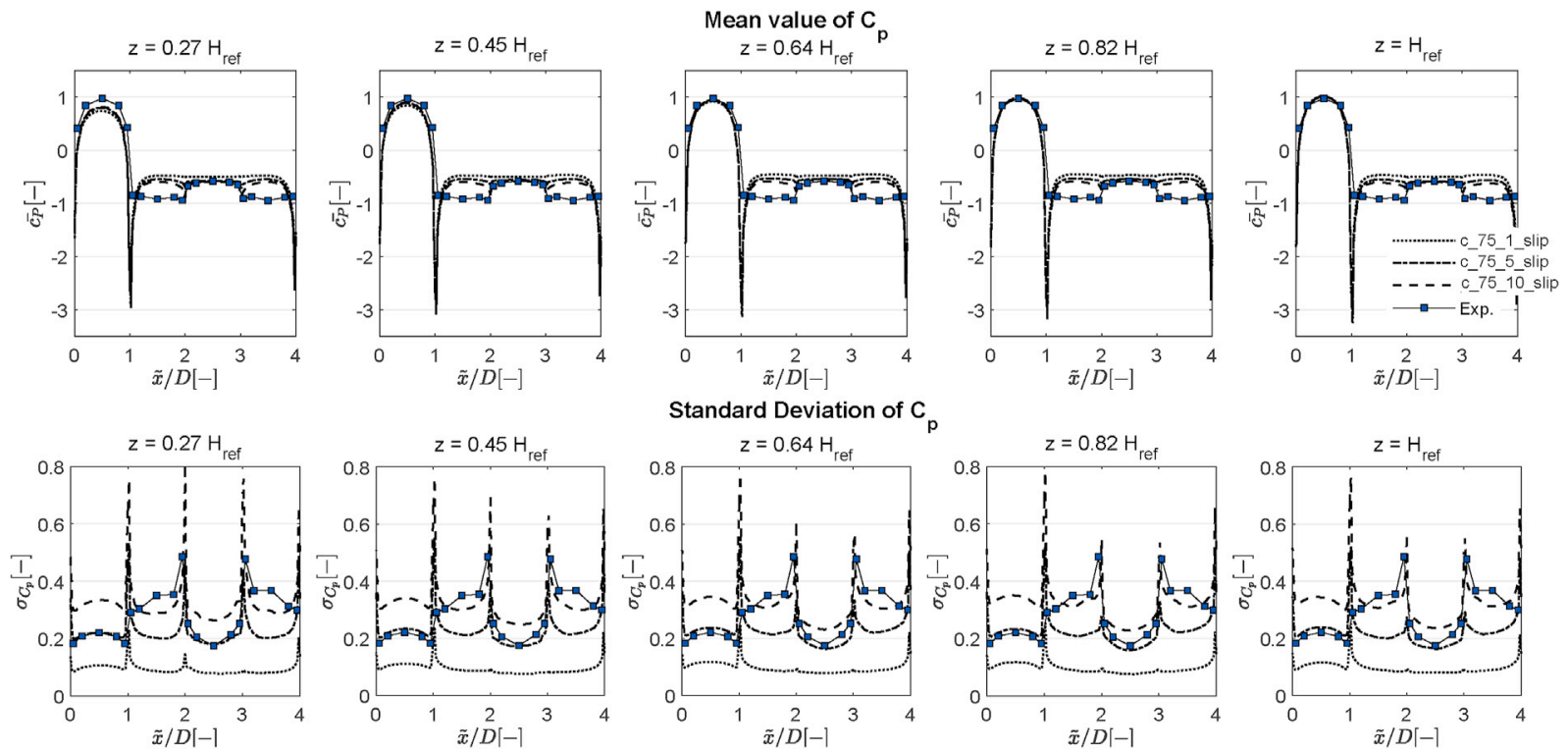

Fig. 19. Effect of the increasing of the turbulence intensity on the pressure statistics.

addressed. Two main differences must be handled between LBM-SEM and the classical SEM for Navier-Stokes equations. First, SEM leads to the definition of a velocity field, while LBM numerical unknowns are the distribution functions. Therefore, a way to reconstruct the later from the SEM velocity field must be chosen. The second problem is that almost all existing SEM models have been developed for incompressible flows, while LBM is related to weakly compressible flows, including the acoustic field, with a variable density. Therefore, an additional hypothesis must be introduced to prescribe density at the inlet plane.

At the initialization a set of $N$ vortices is randomly generated in a virtual box of width $2 L$ downstream the inlet plane. The velocity field on the inlet plane at $t=0$ is then computed following the procedure describe in Section 2. The $N$ vortices are then passively advected at each time step $\left(x_{i}(t+d t)=x_{i}(t)+U^{c} d t\right)$ until they completely cross the inlet plane leading to $x_{i}>x_{0}+L$ with $x_{0}$ the inlet plane location. When the $i$-th vortex cross the inlet plane, a new vortex is randomly generated on the plane $x=x_{0}-L$. This procedure combined with the one described in Section 2 allows to generate the velocity field at the inlet at each time step.

In the present work two options are considered to compute the density field at the inlet: a varying density condition and a frozen density condition. The first one is implemented by enforcing the condition $\partial \rho / \partial n$ $=0$ at the inlet plane using a second order accurate biased finite difference scheme to compute the normal derivative. The second one consists of a frozen density condition: the density at the inlet plane is fixed at a constant uniform value $\rho\left(x_{0}\right)=\rho_{0}$ which permits to reduce generation of the density fluctuations, and then of pressure waves, at the inlet plane.

Once the macroscopic variables, density and velocity, are known at the boundary nodes, the distribution functions should be estimated. The regularization procedure described in Jacob et al. (2018) is used in order 

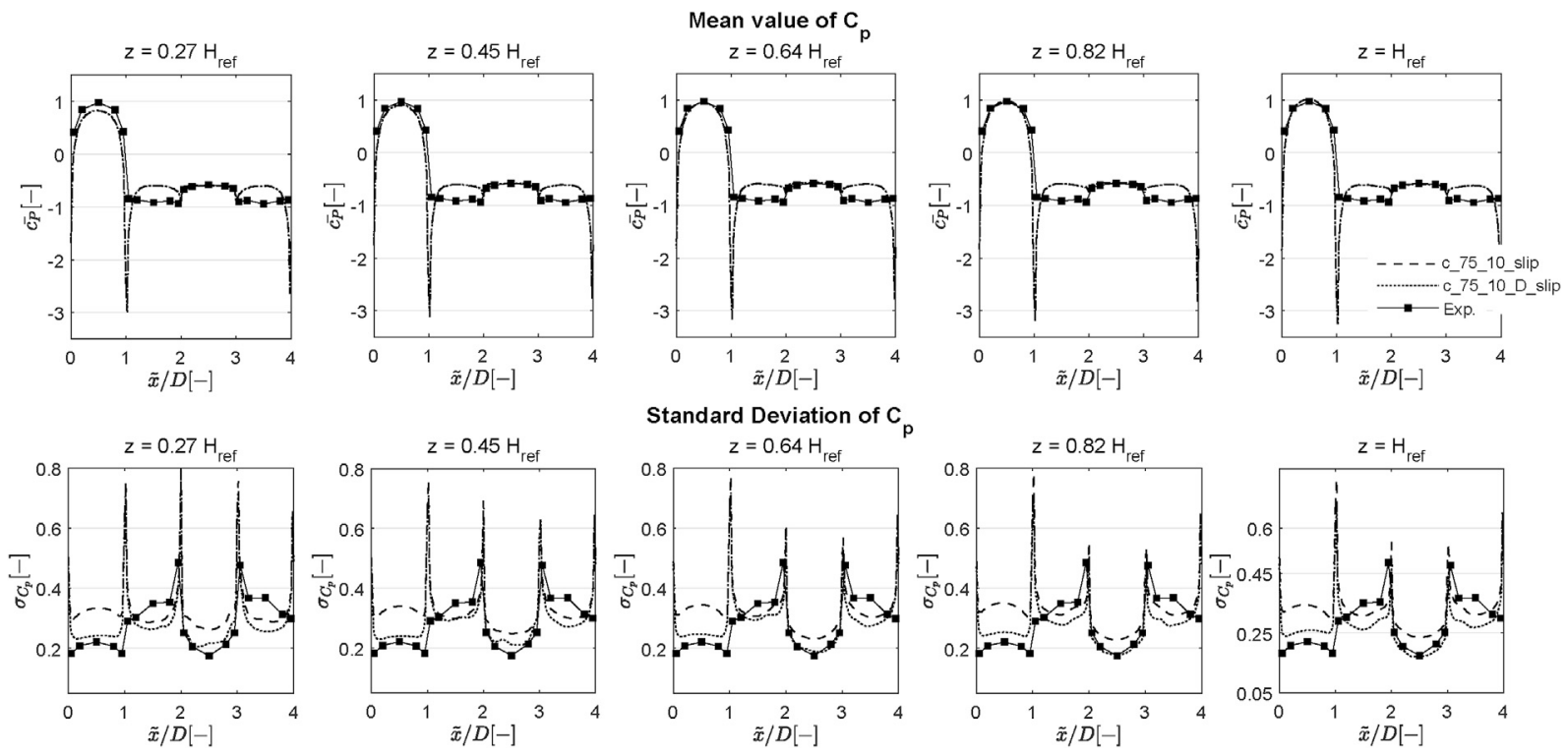

Fig. 20. Mean and standard deviation of pressure coefficients on the tower's sides with varying (c_75_10_slip) and frozen (c_75_10_D_slip) density at the inlet section.
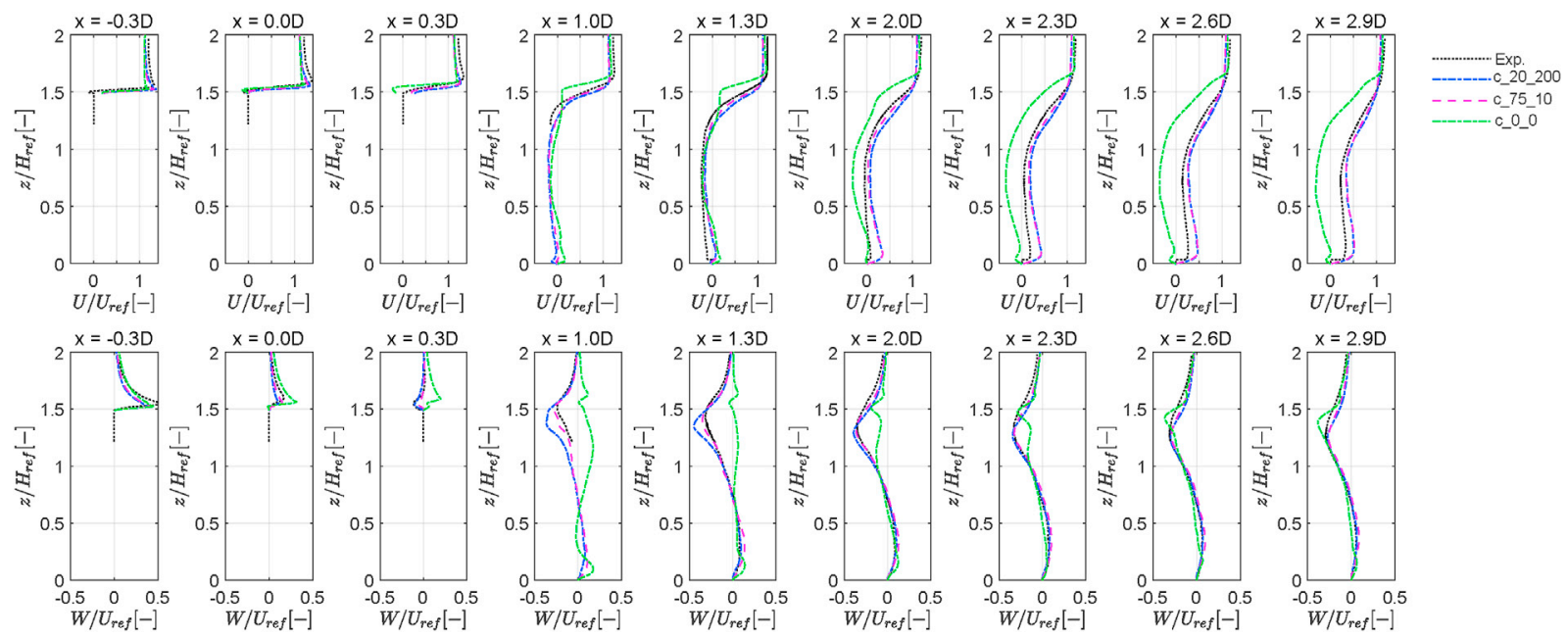

Fig. 21. Streamwise (U) and vertical (W) mean velocity component, in case c_20_200_slip and c_75_10_slip. In green the same profile without the turbulent inlet (case c_____slip). (For interpretation of the references to color in this figure legend, the reader is referred to the Web version of this article.)

to reconstruct the equilibrium and non equilibrium distribution functions following this procedure:

1. Density is computed using frozen density condition $\left(\rho\left(x_{0}\right)=\rho_{0}\right)$ or zero value of the normal gradient $(\partial \rho / \partial n=0)$,

2. Velocity components are computed using Eq. (7),

3. Velocity gradients are computed using finite difference schemes,

4. Equilibrium distribution functions $f_{i}^{e q}(\rho, U)$ are computed from density and velocity values:

$f_{i}^{e q}=\rho \omega_{i}\left[1+\frac{c_{i k} u_{k}}{c_{s}^{2}}+\frac{1}{2 c_{s}^{4}} Q_{i k j} u_{k} u_{j}\right]$

where $c_{s}$ is the speed of sound, $\omega_{i}$ are the weighting coefficients of the D3Q19 lattice and $Q_{k i j}=c_{k i} c_{k j}-c_{s}^{2} \delta_{i j}$. The non equilibrium distribution functions $f_{i}^{\text {neq }}(\rho, \nabla U)$ are computed from density and velocity gradients:

$f_{i}^{n e q}=-\frac{\tau \rho \omega_{i}}{2 c_{s}^{2}} Q_{i k j}\left(\frac{\partial u_{j}}{\partial x_{k}}+\frac{\partial u_{k}}{\partial x_{j}}\right)$ where $\tau$ is the relaxation time used in the LBM collision model.

5. Distribution functions are finally estimated from equilibrium and non equilibrium distribution functions $\left(f_{i}=f_{i}^{e q}+f_{i}^{n e q}\right)$

More details about the boundary treatment are given in Wilhelm et al. (2020) for athermal flows and in Feng et al. (2019c) for thermal flows.

\section{Flow configurations and numerical setup}

\subsection{Description of the grid configurations}

The present work aims at reproducing wind tunnel experiments described in Sheng et al. (2018). The case considered is a square-base tower of original height $147 \mathrm{~m}$; the dimensions of the scaled model are $H=0.49 \mathrm{~m}$ for the height and $D=0.1 \mathrm{~m}$ for the base. The reference height is set at $\sim 2 / 3$ of the total height such as in the experiment, thus $H_{\text {ref }}=0.33 \mathrm{~m}$. The speed at $H_{\text {ref }}, U_{r e f}=10 \mathrm{~m} / \mathrm{s}$, is taken as reference velocity. Consequently, the Reynolds number based on the base $D$ is the same for both the wind tunnel test and the LBM simulation with a value 


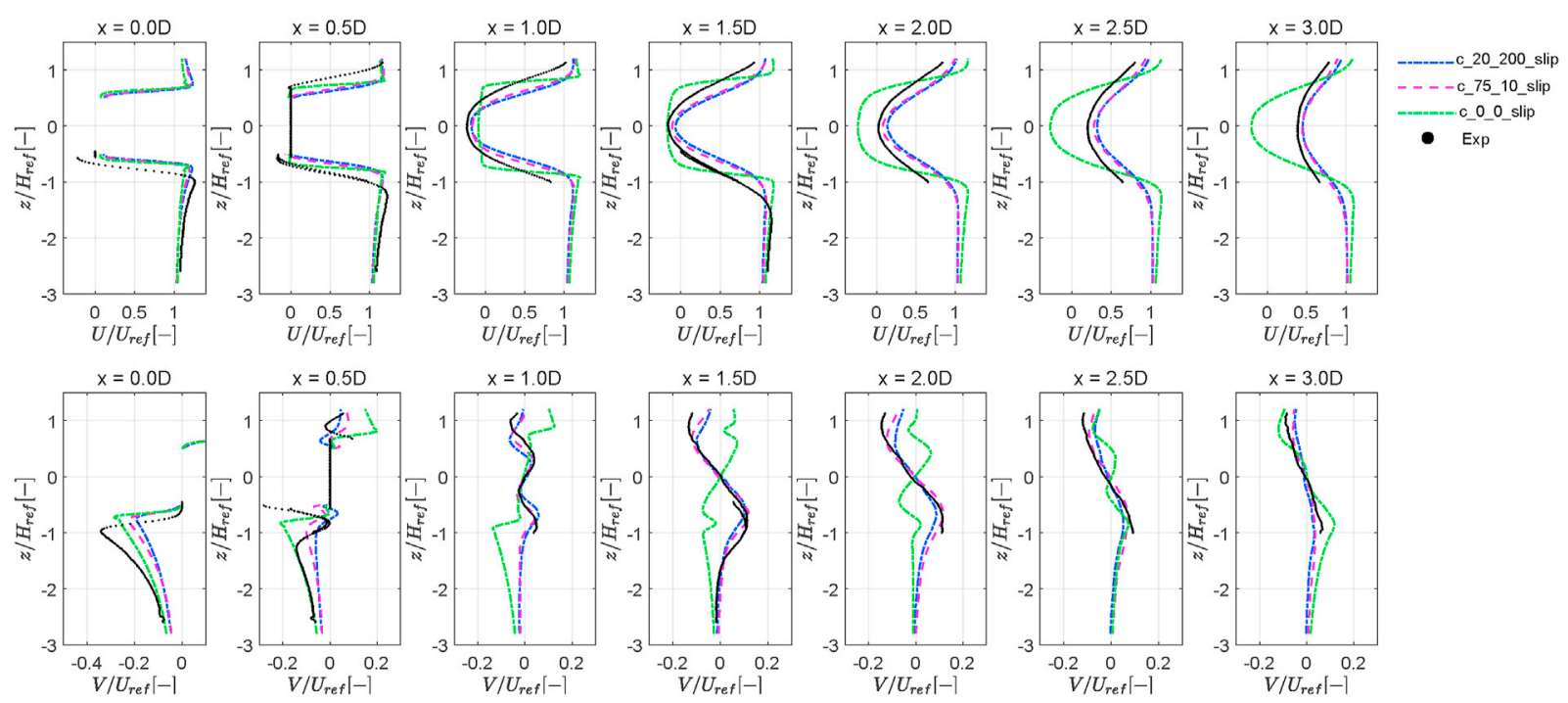

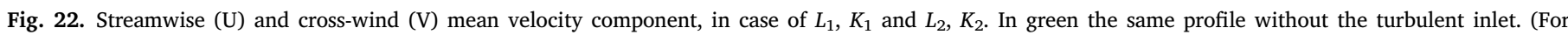
interpretation of the references to color in this figure legend, the reader is referred to the Web version of this article.)
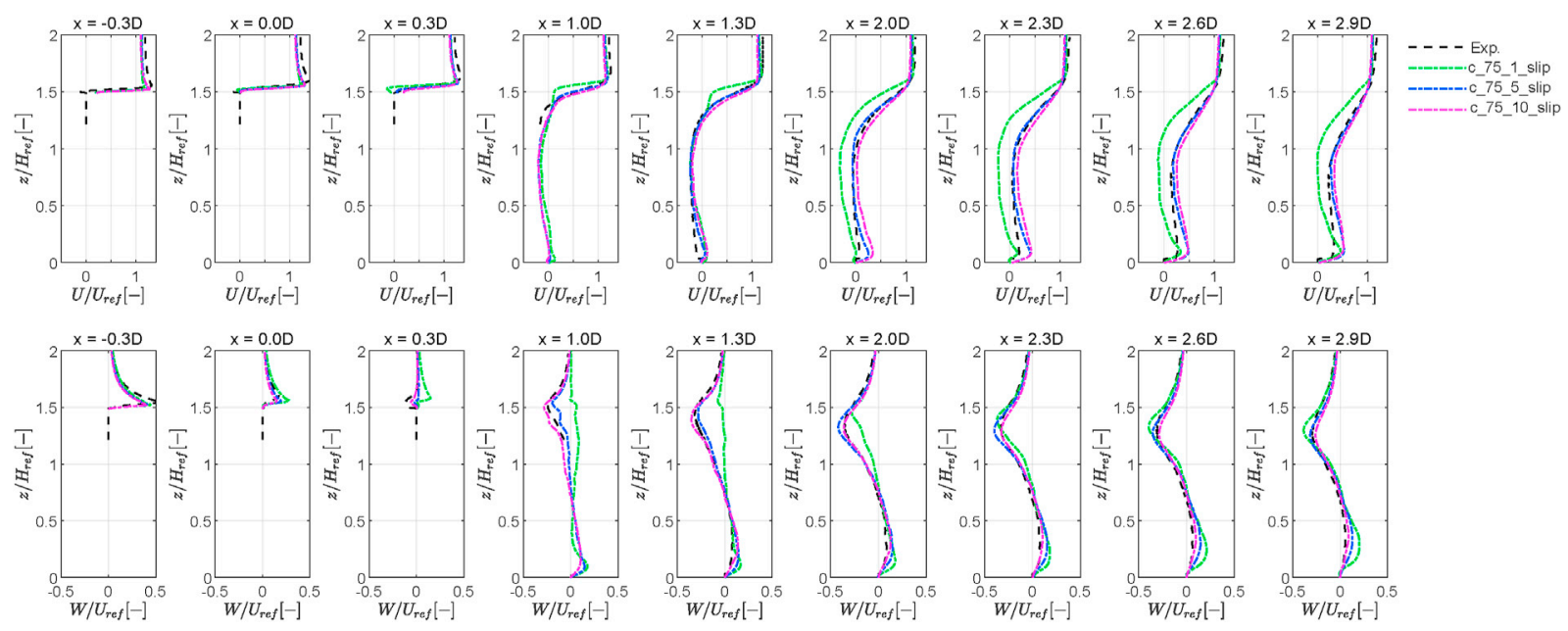

Fig. 23. Streamwise (U) and vertical (W) mean velocity components in the symmetry plane, comparing increasing inlet turbulence intensities.
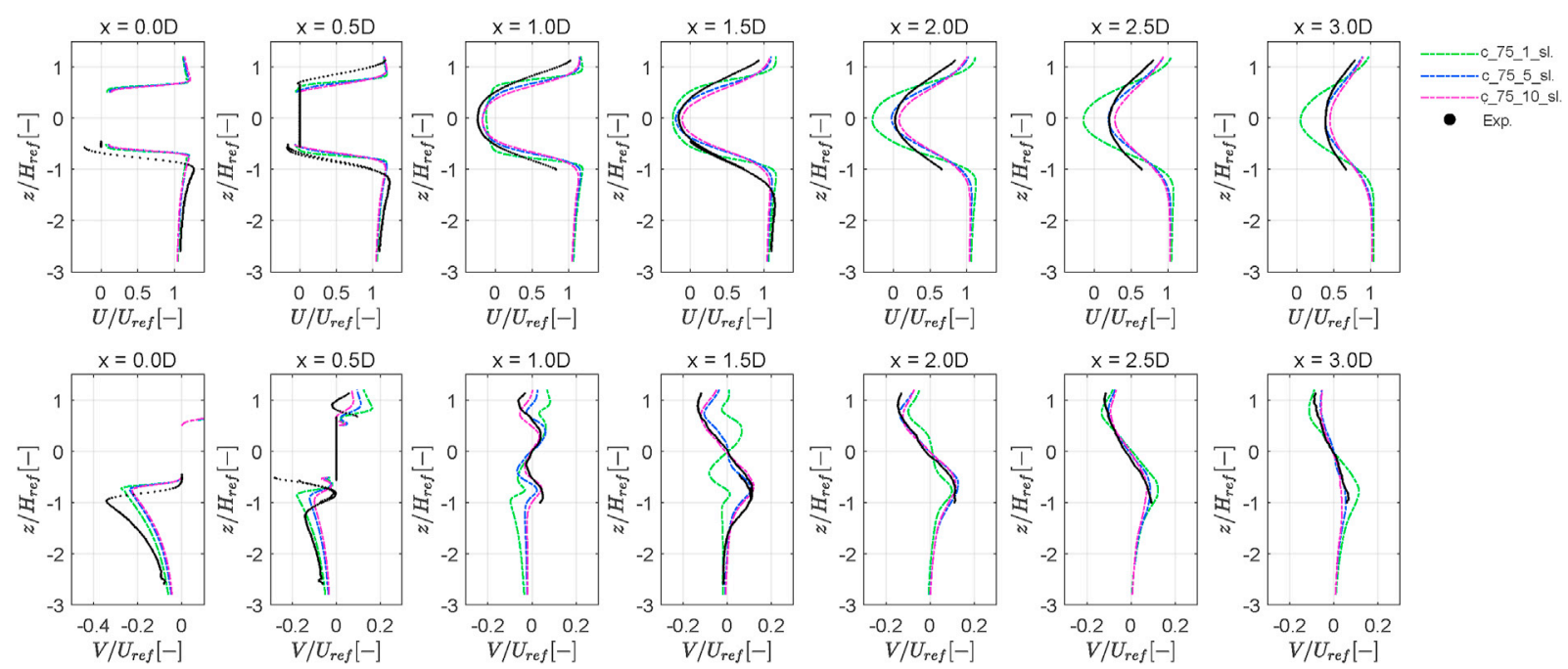

Fig. 24. Streamwise (U) and cross-wind (V) mean velocity components in the horizontal plane at $z=H_{\text {ref }}$, comparing increasing inlet turbulence intensities. 

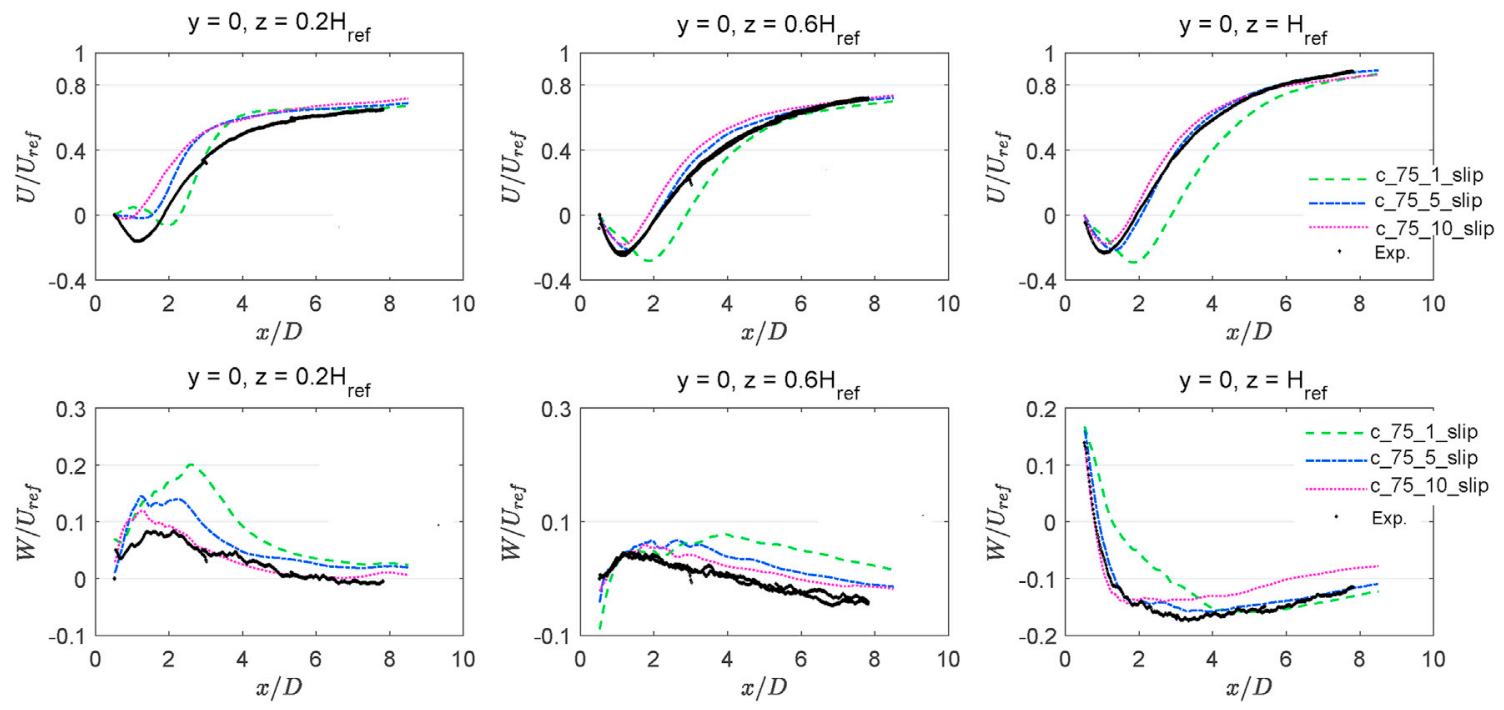

Fig. 25. Streamwise (U) and vertical (W) mean velocities in the symmetry plane along the wake at different heights, comparing increasing inlet turbulence intensities.

of

$\operatorname{Re}=\frac{D U_{\text {ref }}}{\nu_{\text {air }}}=200,000$

Finally, the length scale is thus $\chi_{x}=1 / 300$, the speed scale $\chi_{u}=0.42$ and the time scale $\chi_{t}=\chi_{x} / \chi_{u}=8 \times 10^{-3}$.

The set-up of the domain follows the guidelines given by the Architectural Institute of Japan (Tominaga et al., 2008) and the European Cooperation in Science and Technology (Franke et al., 2007). As detailed in Sheng et al. (2018), the wind tunnel dimension were $20 \mathrm{~m}$ in streamwise dimension, $4 \mathrm{~m}$ width and $2 \mathrm{~m}$ height, being in dimensionless form $40 \mathrm{H}, 8 \mathrm{H}$ and $4 \mathrm{H}$ respectively. The computational domain size are chosen to be $14.5 \mathrm{H}$ in streamwise direction $(4.5 \mathrm{H}$ upstram the building and $10 \mathrm{H}$ downstream the building), $10 \mathrm{H}$ in the crosswind direction and $4 \mathrm{H}$ in height as shown in Fig. 1. According to Tominaga et al. (2008) and Franke et al. (2007), these domain's sizes are sufficient to avoid side effects. Based on these dimension, the blockage ratio in the CFD configuration was

$b \%=\frac{H \times D}{A_{\text {domain }}} \cdot 100=0.51 \%$

with $A_{\text {domain }}$ the cross section of the domain. This value is in agreement this recommendation of Tominaga et al. (2008).

As explained in Section 3.2, embedded subdomains of uniform cubic cells with a ratio of two between two successive subdomains are employed to discretise the computational domain. In the present work 3 different grids were used, one for the free flow simulation used to calibrate the turbulent inflow and two for the simulation of the flow around the building with a coarse and a fine grid. The location of the different subdomains and the grid generated around the building are shown in Fig. 2. It should be noted here that for the free flow simulation (without the building), 3 different refinement levels are used (subdomains represented with black lines in Fig. 2(a)). For the simulation of the flow around the building, 6 refinement levels are used (black and green lines in Fig. 2(a)) in the coarse grid and 7 refinement levels are used in the fine grid (black, green and red lines in Fig. 2(a)).

The largest grid size in all the simulation is defined in non dimensionnal form as $\Delta x_{\max }^{c^{*}}=\Delta x_{\max }^{c} / D=0.5$, leading to a smallest non dimensional grid size of $1.25 \times 10^{-1}, 1.56 \times 10^{-2}$ and $7.81 \times 10^{-3}$ for the free flow, coarse grid and fine grid simulation respectively. These different parameters are summarised in Table 2. For all the presented simulation, an initialization time equal to two flow through times is considered before computing the different statistics in order to be more conservative, even if just one would be sufficient (Pamiès et al., 2009).

\subsection{Description of the boundary conditions considered}

First of all, the boundary conditions used for the free stream simulation are described. This configuration is used to assess the turbulent inlet flow and to analyze the main features of the turbulence advected from the inlet plane. Since the features of upstream turbulence have a deep impact on the interaction with the immersed bluff body, it is important to check the quality of the generated turbulence at the place where the model will be located in the next set of simulations (Vasaturo et al., 2018; Lamberti et al., 2018; Munoz-Esparza et al., 2015). It is reminded here that for this configuration, only 3 mesh refinement levels are considered $\left(\Delta x_{\min }=1.25 \times 10^{-2} \mathrm{~m}\right)$ since there is no need to refine the grid to capture boundary layer dynamics at the surface of the building. They all start from the inlet section (Fig. 2(a)) in order to carry the flow up to the building location without excessive turbulence dissipation. According to the SEM, both the mean velocity profile and the turbulence parameters should be set at the inlet section. For the former, following the recommendations given in Sheng et al. (2018) we use an Eurocode profile (Eurocode, 2005) with a roughness length $z_{0}=0.02 \mathrm{~m}$, which corresponds to an intermediate class between I and II. The mean wind profile reproduced in the scaled system is thus:

$U(z)=\chi_{u} \cdot U_{b} \cdot k_{r} \ln \left(z^{*} / z_{0}\right) \cdot c_{0}$

$z^{*}=\left\{\begin{aligned} z_{\max } & \text { if } z / \chi_{x} \geq z_{\max } \\ z_{\min } & \text { if } z / \chi_{x} \leq z_{\min } \\ z / \chi_{x} & \text { elsewhere }\end{aligned}\right.$

where $U_{b}=16 \mathrm{~m} / \mathrm{s}$ is the effective mean velocity at $z_{\text {fullscale }}=10 \mathrm{~m}$ in full scale, $c_{0}$ is the orography factor $(=1)$, and $z$ is the vertical coordinate in the scaled system $\left(z=\chi_{x} \cdot z_{\text {fullscale }}\right)$. The other variables $k_{r}$ (terrain factor), $z_{\min }$ and $z_{\max }$ are defined in Eurocode and its French Country Annex.

For the turbulence parameters (Pamiès et al., 2009), we focus on the choice of the number of spots $N$, the turbulence length scale $L$ and a corrective factor $K$, which rescales the Reynolds stresses at the inlet section. The combinations of inlet parameters considered in this paper are summarised in Table 3.

The other main parameters are the Reynolds stresses, which are given experimentally. Their diagonal components are derived from the turbulence intensities, being, for instance, $I_{u}^{2}=u^{\prime} u^{\prime} / U^{2}$, where $u^{\prime}(t, x)=u(t$, $\boldsymbol{x})-U(\boldsymbol{x})$ is the oscillating part of the velocity component $u$ and $U$ is the streamwise mean velocity (the same reasoning is made for $v$ and $w$ ). The 
streamwise turbulence intensity is given by the Eurocode:

$I_{u}(z)=\frac{\sigma_{u}^{\prime}}{U(z)}=\frac{k_{I}}{c_{0} \ln \left(\frac{z^{*}}{z_{0}}\right)}$

Where:

$k_{I}=\left[1-2 \cdot 10^{-4}\left(\log \left(z_{0}\right)+3\right)^{6}\right] c_{0}$

On the other hand, the crosswind and vertical turbulence intensities measured in the wind tunnel (Sheng et al., 2018) and fitted to:

$I_{v}(z)=\frac{0.88}{\ln \left(\frac{z}{2.5 \cdot 10^{-5}}\right)} \quad I_{w}(z)=0.08$

were adopted.

Successively, the respective simulations with the tower are verified for the most significant inlets profiles. We remind here that for the building cases, 3 or 4 additional refinement levels are considered around the tower, for a coarser and finer grid (Fig. 2). The mesh size at the surface of the building model is $\Delta x / D=1.56 \times 10^{-2}$ and $\Delta x / D=7.81 \times$ $10^{-3}$ on the fine and coarse grids, respectively, where $D$ is the base of the model. This corresponds to 64 and 128 cells along the base of the model, taking into account that in the present off-lattice implementation of boundary conditions solid walls are located inside computational cells.

The different parameters considered in the building simulations for 1) the SEM parameters (L and K), 2) the density treatment at the inlet and 3) the treatment of building walls are summarised in Table 4. These different sets of parameters will permit here to assess the influence of the inlet condition on the flow around the building, but also the influence of the wall treatment on the building considering 1) frictionless wall with a slip condition, 2) no-slip wall and 3) a turbulent wall model adapted to flows with pressure gradient and separation.

\section{Free stream flow without obstacle}

In this section, the computed mean velocity profile, the turbulence intensities and the power spectral densities are compared with the experimental data, in order to determine the correct inlet parameters. The values are taken at the virtual building location $(x=0, y=0)$.

As expected, the mean velocity profile is not affected by the turbulence parameters and follows well the target imposed at the inlet (Fig. 3).

Looking at the turbulence intensities, they are not influenced by the number of spots N (Fig. 4), which can consequently be chosen quite arbitrary, but at least equal to $N=S_{p} / S_{s}$, where $S_{p}=W \times H$ is the support size (in our case the inlet section) and $S_{s}=4 L_{x} L_{y}$ is the turbulent structure's transverse section (Pamiès et al., 2009). In the present work, the largest value $(N=150)$ is finally chosen, because it seems to reduce the risk of instabilities at the inlet section, especially in correspondence of mesh transitions.

Based on the integral length scales measured in the experiment, two main values are considered for the parameter $L_{1} L_{1}=0.25 \mathrm{~m}$ and $L_{2}=$ $0.75 \mathrm{~m}$. As described in Section 4, different values are tested for the parameter $\mathrm{K}$ which rescales the Reynolds stresses at the inlet section, in order to balance the turbulent energy loss along the path and to recover the targeted flow at the model location. From this parametric study presented in Fig. 5, a specific value of $K$ permitting to recover the target turbulent intensities is found to be respectively $K_{1}=200$ and $K_{2}=10$ for the two different length scale $L_{1}$ and $L_{2}$.

The power spectra of the streamwise and cross-wind oscillating velocity's components are shown in Fig. 6 and compared with experimental data. The Karman function for $u^{\prime}$ and $v$ computed as

$\operatorname{Karm}_{u}(f)=\frac{4 \sigma_{u}^{2} L}{U_{\text {ref }}\left(1+70.8\left(f L / U_{r e f}\right)^{2}\right)^{5 / 6}}$
$\operatorname{Karm}_{v}(f)=\frac{4 \sigma_{v}^{2} L\left(1+188.4\left(2 f L / U_{r e f}\right)^{2}\right)}{U_{r e f}\left(1+70.8\left(2 f L / U_{r e f}\right)^{2}\right)^{11 / 6}}$

is also shown in this figure. It can be seen in Fig. 6 that the first setup with $K_{1}=200$ introduces noise at high frequencies in the power spectral densities of the fluctuating velocity components $u^{\prime}$ and $v^{\prime}$. The indicated cut off frequency is estimated from the local grid size, considering that 10 grid point are needed to capture turbulent structure leading to a cut off Strouhal number of 0.8 . The shorter simulation time with respect to the experimental recordings, causes the PSD to loose the low frequency content; the cut-off high-frequency is set instead by the mesh resolution. However, the typical bell-shape, which is characteristic of a turbulent wind, is already visible and quite in agreement with the experimental data. From this parametric study, we can defined here that the more adapted set of parameter $(\mathrm{N}, \mathrm{L}, \mathrm{K})$ permitting to recover the right turbulent properties in the present case in term of statistics and spectral content is $(150,0.75,10)$.

To reduce pressure oscillations induced by the turbulence generation at the inlet section, a uniform Dirichlet condition on the density is set at this section: $\rho_{\text {inlet }}=\rho_{\text {ref }}$. This prevents the inlet flow to generate acoustic pollution that can negatively affect the correct prediction of pressure fluctuations in case of an incompressible fluid. This possible spurious effect tied to the compressibility of the synthetic turbulence fluctuations has also been reported by several authors in the Navier-Stokes framework, who proposed improved, divergence-free turbulence generation methods (Kondo et al., 1997; Deck et al., 2018; Huang et al., 2010; Gea-Aguilera et al., 2017; Yu and Bai, 2014; Kim et al., 2013; Daniels et al., 2013; Lamberti et al., 2018). In the present case, since LBM is related to a weakly compressible flow, a purely solenoidal inlet condition will not be appropriate. Therefore, it is chosen to freeze density perturbations at the inlet to minimize the radiated noise. Using this frozen-density condition, it is observed that the Lattice-Boltzmann-based Synthetic Eddy Method yields a very satisfactory recovery of the free-stream flow at the model location (Fig. 7).

\section{Flow around an isolated high-rise building: LBM-LES results}

After the inlet flow assessment, the wind's impact on the building is analyzed in the configuration with an incidence angle $\theta=0^{\circ}$ between the incoming flow and the tower front side.

The present section is dedicated to the comparison of the simulation case c_75_10_D_noslip corresponding to the case with $(\mathrm{N}, \mathrm{L}, \mathrm{K})=$ $(150,0.75,10)$ with dirichlet density condition at the inlet and no slip condition on building walls with experimental data. This simulation case is the present reference simulation based on the combination of the most efficient elements. It will be used in Section 7 to investigate the sensitivity of the results with respect to the inlet turbulence reconstruction. For the sake of brevity, the statistical convergence analysis for global forces and pressure coefficients is provided in A, the spectral analysis of wall pressure coefficients is detailed in B and sensitivity to boundary conditions on the building surface and to the grid resolution is analyzed in $\mathrm{C}$.

\subsection{Global forces}

The global forces components $F_{x}, F_{y}$ and $F_{z}$, respectively in streamwise, cross-wind and vertical direction, are considered in their nondimensional form $C_{F i}=F_{i} /\left(0.5 \rho U_{r e f}^{2} D H\right)$. Their averaged values predicted in the simulation are $C_{F_{x}}=1.430, C_{F_{y}}=0.009$ and $C_{F_{z}}=0.191$, while the experimental ones are $C_{F_{x} \text { exp }}=1.271, C_{F_{y} \text { exp }}=0.022$ and $C_{F_{z}, \exp }=0.210$. Looking at $C_{F_{y}}$, numerical results seem more reliable, since it is closer to zero than in the experiment, while it should be theoretically null for $\theta=0^{\circ}$. This slight deviation could be caused by a slight misalignment of the tower in the experimental setup, which may 
also affect the other components. The overall quality is satisfactory, and it is worth noting that analysis of global forces is not presented in many articles related to prediction of wind loads on building.

Looking at the power spectral densities, a very good agreement is also visible (Fig. 8), especially in the prediction of the peak at $S t=0.1$ for $C_{F_{y}}$, caused by the occurrence of vortex shedding.

\subsection{Wall pressure fluctuations on the building}

Such as for the global forces, also local pressures on the tower's side are considered in their non-dimensional form. The pressure coefficients are computed as $C_{p}(t)=\frac{p(t)-p_{\text {ref }}}{q}$ where $p(t)$ is the instantaneous local pressure, $p_{\text {ref }}$ is the static reference pressure and $q=0.5 \rho U_{r e f}^{2}$ is the dynamic pressure of the incoming flow, being $\rho$ the density of the fluid $\left(\rho_{\text {air }}=1.2\right.$ $\mathrm{kg} / \mathrm{m}^{3}$ ).

In the experiment, pressure was recorded with 265 pressure probes placed at 12 different heights all around the perimeter (see Fig. 1) and the remaining on the top facade. The acquisition frequency was $f_{s}=512 \mathrm{~Hz}$, which is the same employed in the sampling of instantaneous pressure in the present simulations, for the computation of the standard deviation $\sigma_{C_{p}}=\sqrt{\left(C_{p}(t)-C_{p}\right)^{2}}$ and the PSDs. This means an acquisition every 130 time steps with a minimum time step of $\Delta t_{\min }=1.504 \times 10^{-5} s$ (for the coarser mesh). Conversely, the mean values are computed directly during the simulation, thus considering all the values at every integration time step.

First and second order statistics of pressure coefficient are computed and compared to experimental values in Fig. 9 at different heights. A very good agreement is observed at all locations for the mean $C_{p}$ value. The computed standard deviation is also reliable, since it recovers all physical trends, especially the vertical variations of the maximum amplitude of the standard deviation. The quality of the present results is similar to those of the best results obtained with advanced Navier-Stokes simulation tools (Daniels et al., 2013; Elshaer et al., 2016).

Fig. 10 shows the color maps of mean value and standard deviation of the pressure coefficients, for both the c_75_10_D_noslip simulation case obtained with the LBM-LES method and the experiment. It is seen that the mean pressure field is recovered in a very accurate way on all sides of the building, and the topology of the mean pressure distribution is well recovered on all sides. The topology of the rms pressure is also correctly predicted, but with a larger error on the amplitudes. The symmetrical pressure distribution is evident looking at the mean value, especiallly in the numerical case; however both the rms show an asymmetry on the lateral facades: this may be caused, for the numerical case, by a limited acquisition time, since second order statistical quantities are more susceptible to time variations than first order ones.

At last, an analysis of the peak values is carried on and compared with experimental values. Based on a simplified observed peak method, the time history is divided into 10 subsets, and then the peak value is estimated as the average of the maximum value in each subset. Both positive and negative peaks $\left(\hat{C}_{p, \max }, \hat{C}_{p, \min }\right)$ are estimated; the latter are usually the most relevant in civil assessment. A first confidence interval analysis of the predicted pressure extrema shows that the $95 \%$ confidence interval width is less than $10 \%$ of the predicted values, leading to a reliable prediction. The results for some location is shown in Fig. 11, showing an overall very satisfactory agreement. Some discrepancies are observed at taps 10,11 and 13 , i.e. near the back face. $\hat{C}_{p, \max }$ is generally underpredicted, probably due to the lower observation time. The differences at the basement of the tower may be caused by a different ground roughness, as also observed in the power spectral densities, and/or some differences in the experimental and predicted horseshoes vortex system at the foot of the building.

For the sake of brevity, results dealing with the spectral analysis of the wall pressure fluctuations (power spectral density and coherence) are displayed in $\mathrm{B}$.

\subsection{Velocity field around the building and in the wake}

Another important point is the capability of the LBM-LES approach to yield accurate prediction of the velocity field around the building and in the wake as shown in Gousseau et al. (2013). It is worth noting that the capability of predicting accurately the flow around the building is of great importance for some urban-flow-related applications, such as pedestrian wind comfort and pollutant dispersion. The mean velocity fields are compared to experimental PIV data on one horizontal plane (at $z=0.19 H_{\text {ref }}$ ) and in the vertical symmetry plane at $y=0$ in Figs. 12 and 13 , respectively. In all cases a very good agreement is observed between LBM-LES data and experimental data, both on the sides and top of building demonstrating the high accuracy of the simulation in detached areas along building walls and also in the wake region downstream the building.

A deeper insight into the quality of the capture of the turbulence dynamics in the flow is gained looking at the turbulent shear stress $u^{\prime} v^{\prime}$. Comparisons with experimental data in the wake are displayed in Fig. 14, showing the high accuracy of the present LBM-LES solution.

\section{Sensitivity of the results to inlet turbulence reconstruction}

In this section, the sensitivity of the results with respect to the synthetic turbulence put at the inlet plane is investigated considering the cases gathered in Table 4.

\subsection{Sensitivity of global forces}

The first quantities considered here are the global forces and moments, whose mean and standard deviation values are displayed in Tables 5 and 6. Comparing case c_0_0_slip (i.e. the case with steady inflow) with other cases, one can see that inlet turbulence leads to a significant increase of the forces and their moments. This is consistent with previous physical analyses dealing with the effects of upstream turbulence on immersed bluff bodies (Hillier and Cherry, 1981; Blackburn and Melbourne, 1996; Hearst et al., 2016; Castro and Robins, 1977). It can be understood using Gerrard's theory (Gerrard, 1966) for the mechanics of the formation region downstream a bluff body, which states that the vortex shedding mechanisms is mainly driven by the interactions between the two separated shear layers and the entrainment of fluid between the interior of the formation region and the outer flow, leading to the definition of two main length scales, namely the length of the formation region and the diffusion length (defined as the thickness of the shear layer at the end of the formation region). Inlet turbulence leads to an increase of the diffusion length and a decrease of the formation region length, resulting in changes in the mean and fluctuating forces exerted by the wake motion on the solid body. Increasing the rescaling factor of Reynolds stresses at the inlet, $K$, yields higher loads on the building, which is coherent with the fact that a stronger turbulence activity should increase the aerodynamic forces on an immersed body.

However, the influence of the inlet turbulence parameters is more visible in power spectral densities. In Fig. 15 that compares cases c_20_200_slip and c_75_10_slip, the flow has the same turbulence intensities at the inlet, but a different combination of integral length scale and Reynolds stresses. The decrease of the integral scale at the inlet in case c_20_200_slip leads to an expected shift of the peak of the force spectra to higher frequencies. This can be explained by the fact that reducing the size of energetic eddies in the upstream turbulence will lead to a higher frequency forcing of the separated shear layers near the cylinder, leading to the selection of higher frequency modes in their transition process and therefore changing the entrainment rate in the shear layers, the diffusion length and the formation region length. In Fig. 16, which is related to cases c_75_1_slip and c_75_5_slip, the length scale is kept constant, and the Reynolds stresses intensity is lowered. In the case with very low turbulence intensity, i.e. the case c_75_1_slip in which the 
inlet Reynolds stress amplitude has been reduced by a factor $80 \%$, a small secondary peak is observed, which may be tied to the first harmonic of the vortex shedding main frequency. This secondary peak is masked by upstream turbulence in cases with strong inlet turbulence, since inlet fluctuations tend to scramble separated shear layers and coherent motion in the wake by weakening the interactions between the shear layers with opposite vorticity. In order to assess this statement, a simulation without the SEM is carried out (case c_0_0_slip), to check the effective importance of the generation of inlet turbulence (see Fig. 17). Peaks related to harmonics of the vortex shedding main frequency are now clearly observed.

At last, results obtained using the Dirichlet low noise frozen-density condition at the inlet plane along with SEM are shown in Fig. 18. The three considered variants of model for the solid walls on the building (slip wall, no-slip wall and turbulent wall model) are compared to investigate the generality of the conclusion about the inlet noise influence. They all lead to very similar results, which are in better agreement than those shown above with variable density inlet condition: a reduction of noise in high frequency is visible, and the peak is better predicted. A deeper analysis on the effect of building wall treatment is given in $\mathrm{C}$.

\subsection{Pressure coefficients}

The major influence of inlet turbulence on computed wall pressure is observed on the standard deviation, which takes into account the oscillating part of the local pressure. In Fig. 19 the increase of inlet turbulence (i.e. increasing the scaling factor $K$ ) leads to the increase of standard deviation over all the building sides; on the contrary, the mean value is not affected, since it mainly depends on the mean velocity profile. It can be seen on Fig. 20 that the use of a frozen-density condition at the inlet section permits to reduce the acoustic pollution in the flow which lead to a reduction of the pressure coefficient fluctuations on the front wall and a better agreement with experimental data. It is worth noting here that this frozen-density condition has no effect on the mean pressure coefficient on the front wall.

\subsection{Mean flow velocity in the building wake}

The mean velocity fields in the wake are now compared. The planes considered here are the same as those corresponding to the PIV measurements in the experiment. For the sake of brevity, a single horizontal plane $\left(z=H_{\text {ref }}\right)$ is shown for each group of simulations.

Cases c_20_200_slip, c_75_10_slip and c_0_0_slip (steady inlet) are compared in Figs. 21-22. It is seen that cases c_20_200_slip and c_75_10_slip exhibit similar results in good agreement with experimental data, while the steady inlet case c_0_0_slip yields large discrepancies in the topology of the computed flow, with a too large recirculation bubble downstream the building. This is due to the fact that upstream turbulent fluctuations trigger a fast growth of the Kelvin-Helmholtz instabilities in the separated shear layers due to their high receptivity to external disturbances, and therefore to a stronger entrainment mechanism across these shear layers which exhibit a larger spreading rate. These phenomena are associated to a decrease of the formation region length and an increase of the diffusion length. Therefore, one can conclude that the flow topology is sensitive to the fact that some turbulence is injected at the inlet, but not to very fine details of the synthetic turbulence (the integral scale $L$ and the rescaling factor $K$ in the present case). This is also checked by looking at Fig. 23 and 24, which displayed results for cases c_75_10_slip, c_75_1_slip and c_75_5_slip, i.e. by changing the rescaling factor $K$ at fixed turbulence lengthscale $L$. Changing $K$ by a factor 10 yield close results.
Larger discrepancies are seen in Fig. 25, which displays the mean velocity components along lines in the symmetry plane in the wake. As expected, the main influence of inlet fluctuations is to decrease the size of the recirculation bubbles on the side and downstream the building, by augmenting the spreading rate and the entrainment rate of shear layers that develop at the interface between recirculating areas and mean flow. In the case with weak inlet fluctuations (case c_75_1_slip), the recirculation region in the wake is too large, showing that inlet turbulence were not strong enough to ensure a correct transition rate to turbulence in the separated shear layers. (See Fig. 24)

\section{Conclusions}

A Lattice-Boltzmann-based Large Eddy Simulation method for wind load prediction on a high-rise building has been proposed and assessed using a recent very detailed experimental data base. The generation of inlet turbulence via an extension of SEM to the present weakly compressible LBM framework was paid a particular attention. A new lownoise frozen-density implementation of SEM was proposed, which minimizes the amount of spurious pressure waves generated by the fluctuating inlet condition. Validation of the present results was carried out considering a huge amount of physical quantities, both at the building surface and in the wake, leading to an almost unique validation of such a simulation. Using a well suited grid resolution along with a well calibrated Synthetic Eddy Method, very good agreement has been obtained with experimental data at all validation levels, including mean and rms values, spectral analysis and local instantaneous pressure maxima, showing the reliability of the present simulation tool for wind load prediction. A detailed sensitivity analysis was also performed. To the author's knowledge, the present work is among the most detailed available ones dealing with validation of LES for the prediction of wind loads on buildings, providing a deeper insight into the potential of LES for wind engineering.

\section{CRediT authorship contribution statement}

Elisa Buffa: Investigation, Formal analysis, Visualization, Writing review \& editing. Jérôme Jacob: Resources, Investigation, Methodology, Software, Supervision, Writing - review \& editing. Pierre Sagaut: Conceptualization, Methodology, Supervision, Writing - review \& editing.

\section{Declaration of competing interest}

The authors declare that they have no known competing financial interests or personal relationships that could have appeared to influence the work reported in this paper.

\section{Acknowledgements}

Centre de Calcul Intensif d'Aix-Marseille is acknowledged for granting access to its high performance computing resources. This work was performed using HPC resources from GENCI-TGCC/CINES (Grant 2020A0072A07679). This work was also supported by the ProLB software project (http://www.prolb-cfd.com). Dr. Sylvain Aguinaga (CSTB), Dr. Julien Guilhot (CSTB) and Dr. Julien Roge (CSTB) are warmly acknowledged for providing experimental data and fruitful discussions. Prof. Alberto Zasso (Politecnico di Milano) is acknowledged for fruitful discussions and raising the attention on the analysis of the pressure peak values.

\section{Appendix A. Statistical convergence analysis (case c_75_10_D_noslip)}

The convergence analysis of statistical quantities presented in Section 6 for the global force and pressure coefficients on case c_75_10_D_noslip is 
checked and presented hereafter. As convergence time, it is considered that time when the residuals function $\Phi_{\text {res }}$ goes under a threshold (e.g. $\left.10 \%\right)$. The procedure is similar to the one proposed by Bruno et al. (2010) and adopted by Ricci et al. (2018): the time history is divided into $N_{D}$ sub-windows of length $T$; then the average (or the standard deviation) $\varphi_{n}$ is computed over a time $t_{n}=[0, n T]$, where $n=1,2 \ldots N_{D}$. The residuals percentage is computed as:

$\Phi_{\text {res }}^{n}[\%]=\left|\frac{\varphi_{n}-\varphi_{n-1}}{\varphi_{n}}\right| \cdot 100$

In our case $N_{D}$ is set equal to 40 . The time track is considered after an initialization time taken equal to two through-out of the inlet flow, thus in the normalized form $t_{\text {init }}^{*}=t_{\text {init }} \cdot U_{\text {ref }} / D=150$. Looking at Fig. A.26, it is seen that a satisfactory convergence $\left(\Phi_{\text {res }}<10 \%\right)$ is obtained after $t^{*} \simeq 500$.
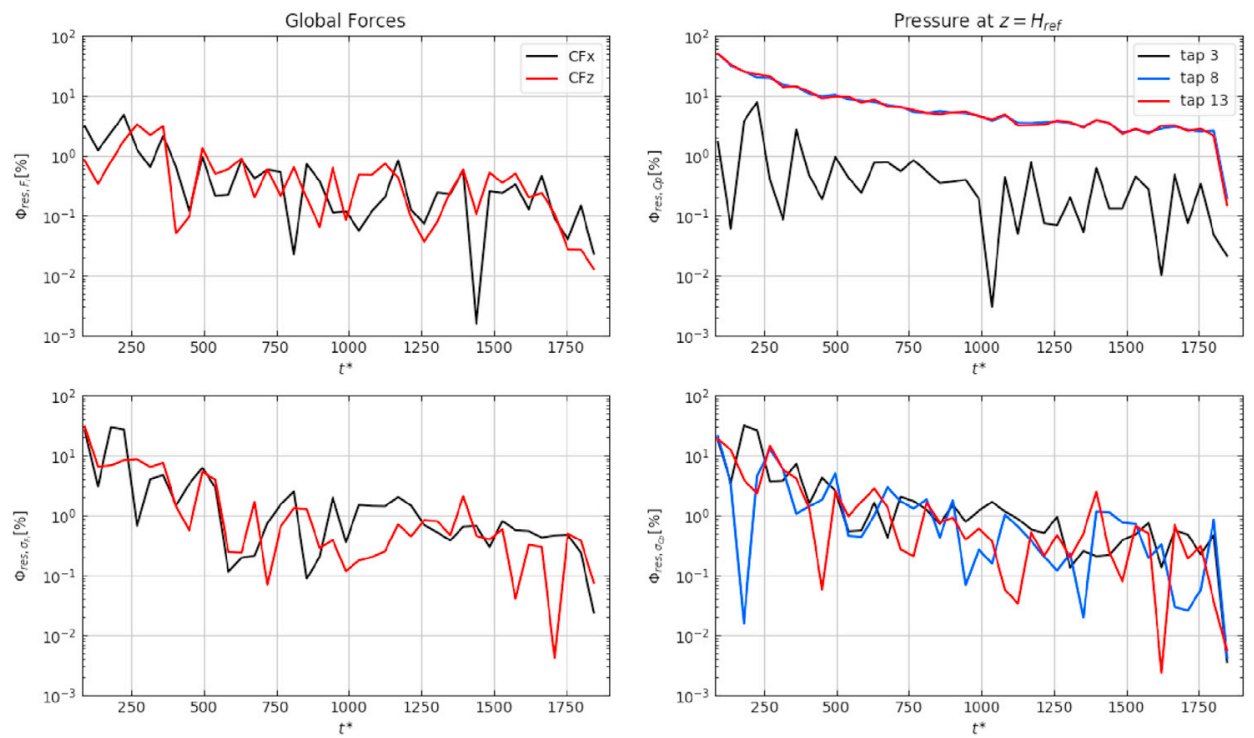

Fig. A.26. Convergence of first and second moments for the drag and lift forces (left) and the pressure coefficients (right). Pressures refers to central points on front, side and back facades at $z=H_{\text {ref. }}$

\section{Appendix B. Spectral analysis of computed wall pressure fluctuations (case c_75_10_D_noslip)}

The frequency content of the wall pressure coefficient presented in Section 6 for case c_75_10_D_noslip are also considered. The PSD of wall pressure measured on the tap location at two different height are plotted on Fig. B.27. A clear peak of pressure is visible at the Strouhal number $S t=f \cdot D / U_{\text {ref }} \simeq$ 0.1 for the points on the lateral sides, in agreement with experimental observations. However numerical simulations do not recover the shift towards higher frequencies of this peak when approaching the floor of the wind tunnel reported in the experiments (see Fig. 13 in Sheng et al. (2018)). This may be caused by an higher effective roughness of the floor in the wind tunnel test, which is instead modeled in the simulation by an arbitrary roughness coefficient in the wall model that do not account for the production of small vortical fluctuations by experimental roughness elements, leading to slight differences in the turbulent features of the near-wall turbulent layer.
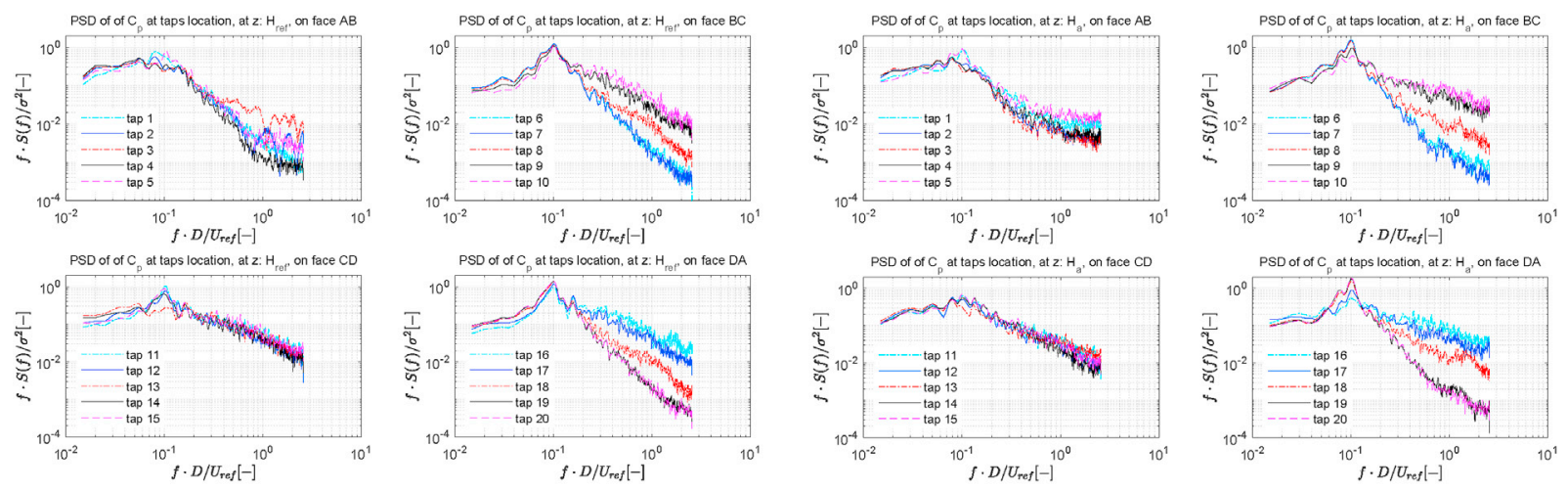

(a)

(b)

Fig. B.27. Power spectral densities of the pressure measured at experimental tap locations at $z=H_{r e f}$ (a) and $z=0.09 H_{r e f}=H_{a}$ (b). The peak of power at the Strouhal number $S t=0.1$ is particularly visible on the lateral sides and near the leeward edges. 
The spectral coherence computed between different taps are also considered in the present study, with the coherence computed as:

$\operatorname{Coh}(f)=\frac{|X Y(f)|^{2}}{X(f) Y(f)}$

where $X Y(f)$ is the estimated cross-spectrum of signals $x(t)$ and $y(t)$, and $X(f)$ and $Y(f)$ are the estimated power spectra of $x(t)$ and $y(t)$ respectively. The coherence highlights the Strouhal number relative to vortex shedding, especially for those points located on the lateral sides and near the edges (Fig. B.28), which are the most involved in this phenomenon.
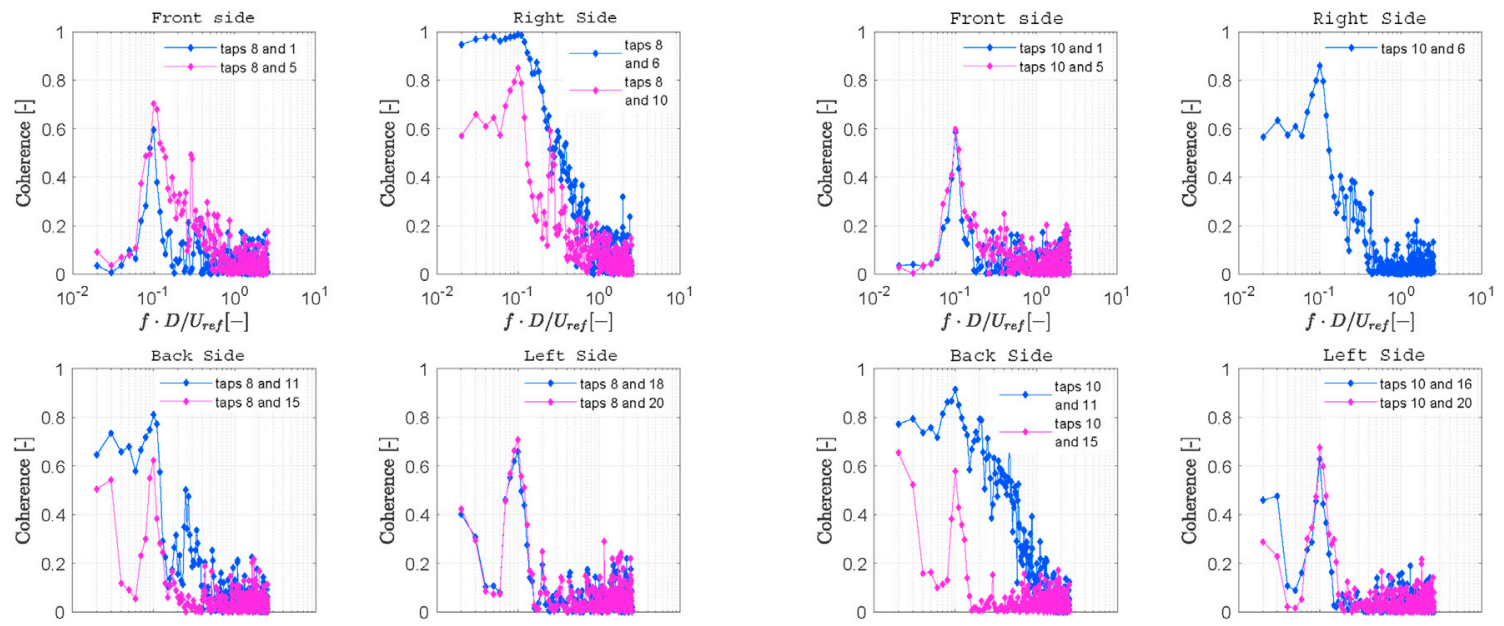

(a)

(b)

Fig. B.28. Coherence at $z=H_{\text {ref }}$ of pressure taps 8 (a) and 10 (b). Especially at opposite lateral side, the coherence is high at $S t=0.1$.

Looking at the coherence between two different heights (between $0.09 H_{r e f}$ and $H_{r e f}$ ) presented on Fig. B.29, it is clear that this phenomenon takes place at almost any horizontal section of the tower, since even between $H_{a}$ and $H_{r e f}$ the peak of coherence holds. These observations are in agreement with experimental findings.
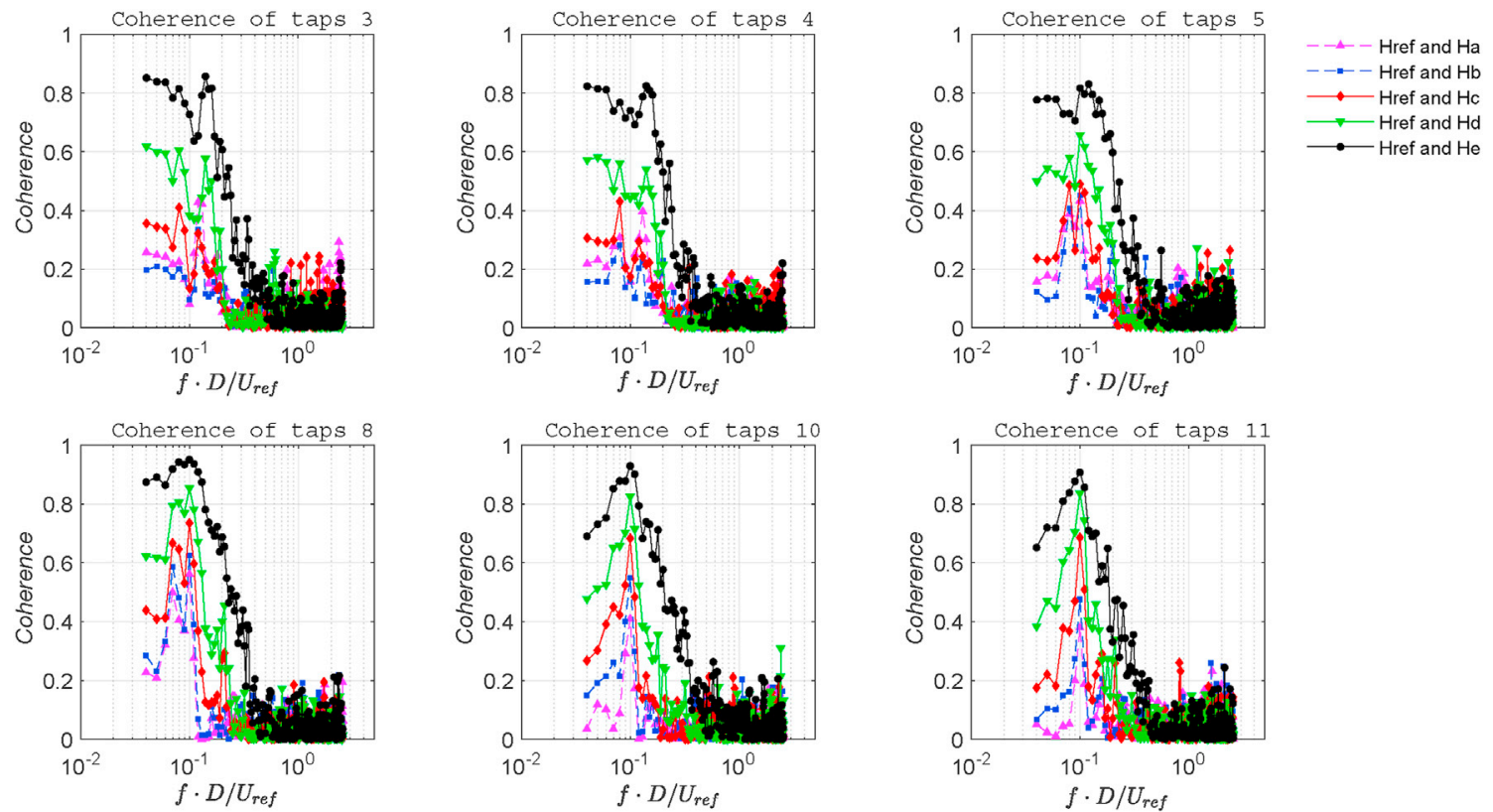

Fig. B.29. Coherence between taps at same longitudinal location but at different heights $\left(H_{a}, H_{b} \ldots H_{r e f}\right)$. $H_{a}=0.09 H_{r e f}$ is the first line of taps and $H_{r e f}$ the sixth in the experimental setup. 
In this section, the sensitivity of the results with respect to i) the boundary condition used at the building surface and ii) the grid resolution is investigated, considering the cases given in Table 4.

\section{Appendix C.1 Global Forces}

Looking at Table 5, the sensitivity of the global forces and moments to the wall model is observed to be significant. The slip wall condition (case c_75_10_D_slip) yields a significant under prediction of the mean forces, which can be explained by a lack of production of vorticity at the wall that significantly modify the wake and the flow in the boundary layers along the building. On the contrary, a no-slip wall condition (case c_75_10_D_noslip) yields an overprediction of the vorticity generation at the wall associated to a too high drag value. While mean values are different, the force spectra are observed to be almost insensitive to the wall boundary conditions (see Fig. 18). This is due to the fact that the most energetic flow fluctuations are governed by large structures in the wake of the building, whose dynamics is governed by vorticity generation at the geometric singularities (building's edges and corners) and the inviscid mechanisms described by Gerrard (1966), namely the spreading of each separated shear layer due to Kelvin-Helmholtz instability and turbulent fluctuations on the one hand, and the interaction between the two shear layers on the other hand.

Grid resolution effects on the global forces is seen comparing cases f_75_10_slip (fine grid) and c_75_10_slip (same wall boundary condition as f_75_10_slip but on the medium grid). It is seen that grid refinement yields a significant improvement of the overall accuracy of prediction of mean forces and moments on the building. This is explained by a better capture of the separated shear layer dynamics on the sides of the building and in the formation region in the wake. This is in agreement with statements dealing with grid resolution effects in large eddy simulation of the flow around a square cylinder at lower Reynolds number given in Ref.(Cao et al., 2020).

\section{Appendix C.2 Wall pressure fluctuations on the building}

Wall boundary conditions significantly affect the local pressure that acts on the towers sides. The main influence is observed on the standard deviation, which takes into account the oscillating part of the local pressure (see Fig. C.30). In this case the Dirichlet no-slip condition on velocity predicts the pressure's statistics better than the turbulent wall law, especially nearby the edges. This might be due to the fact that the Reynolds number is too low in wind tunnel experiments at the 1:300 scale to ensure that turbulence is fully developed in boundary layers and separated regions on the building sides. Therefore, wall models that rely on the assumption that the turbulence is fully developed may loose their efficiency in such a case, especially in massively separated regions in which the Reynolds number based on the recirculating velocity is much smaller than the one based on the upstream velocity. In this case, the no-slip conditions is the most appropriate one.
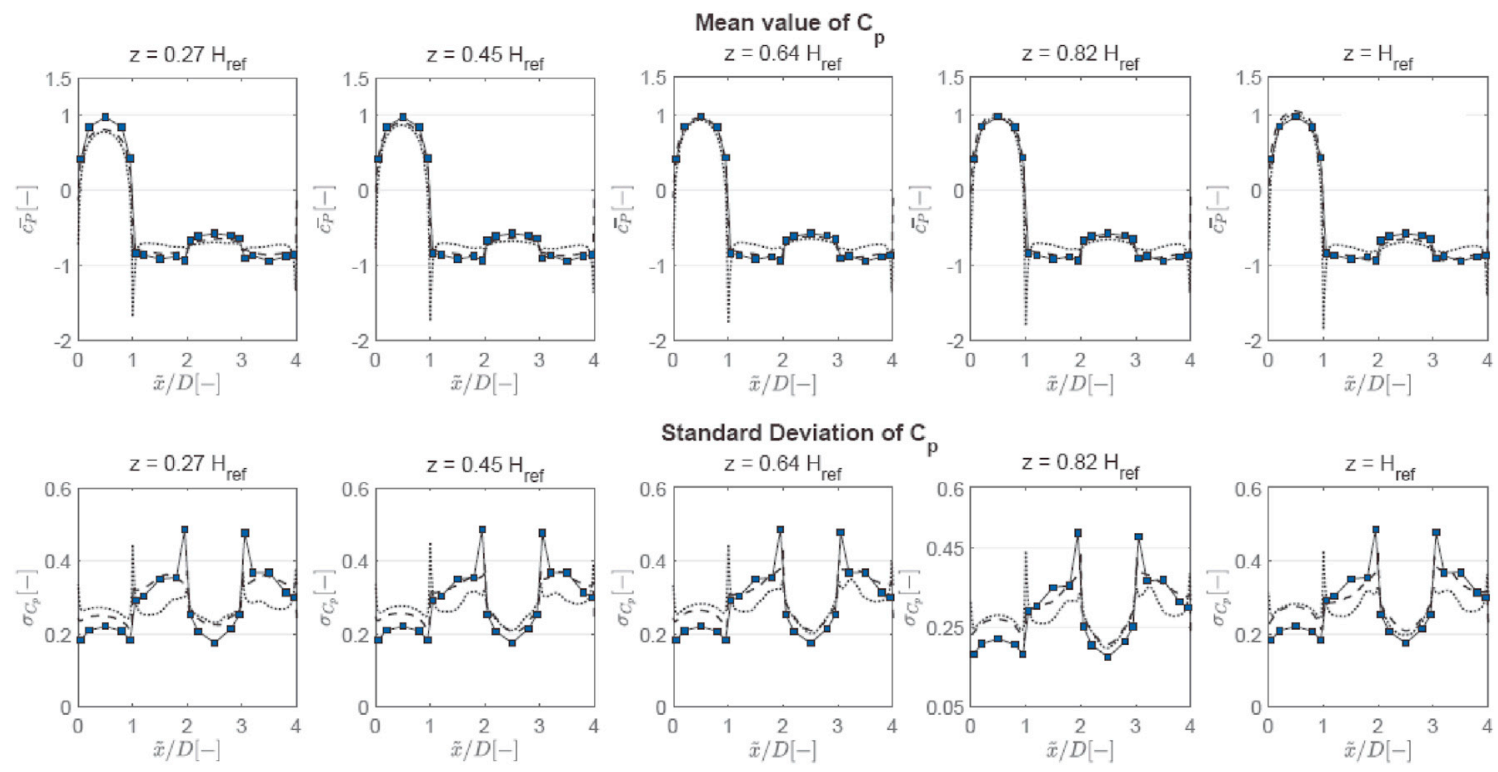

Fig. C.30. Pressure coefficients along the tower's perimeters obtained with no-slip (c_75_10_D_noslip, dashed line) and turbulent wall model (c_75_10_D_model, dotted line) conditions on the building sides.

At last, the effect of mesh refinement is addressed looking at Fig. C.31. Grid refinement leads to a better agreement with experimental data in the prediction of the pressure (both mean value and STD) on the lateral sides, on which very complex separation bubbles occur. However, since it doesn't influence visibly the prediction of the wake, the coarser mesh can be considered to be reliable enough for practical engineering use. 

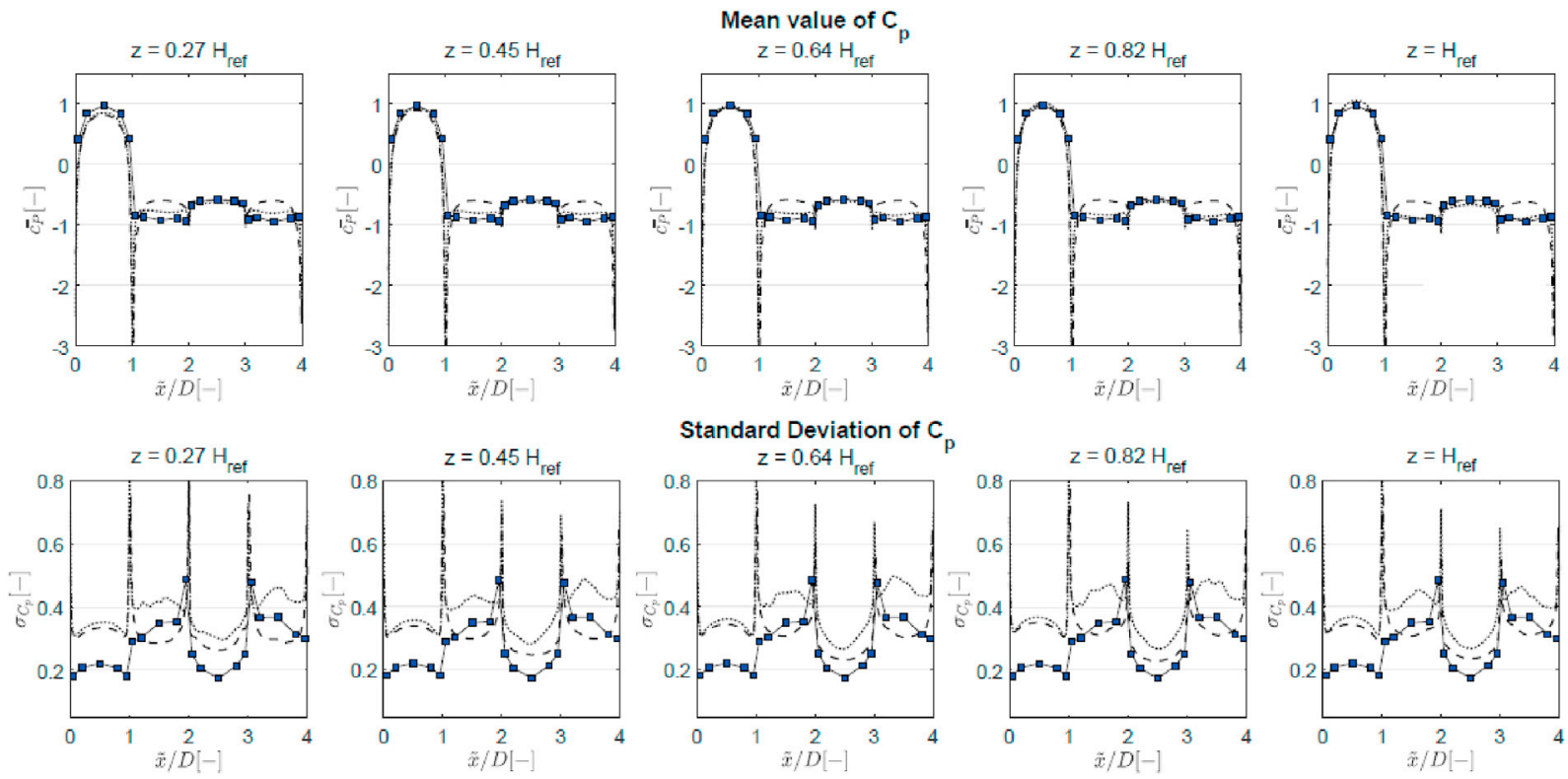

Fig. C.31. Pressure coefficients statistics predicted with coarse (c_75_10_slip, dashed line) and fine (f_75_10_slip, dotted line) mesh.

The influence of boundary conditions used at the building surface is addressed in Figs. C.32 and C.33, that display the mean velocity components for cases c_75_10_D_slip (slip condition), c_75_10_D_noslip (no-slip condition) and c_75_10_D_model (turbulent wall model). It is seen that the slip condition yields an underestimation of recirculation bubbles on the side walls, which is coherent with the lack of production of vorticity at the building surface. In the wake, the sensitivity of the solution is much weaker and all approaches yield results in close agreement with experimental data. This is due to the fact that separation is triggered by building corners, which are singular points at which vorticity is created. A building with circular section on which separation is governed by the balance between turbulence and pressure gradient would have lead to much larger differences.
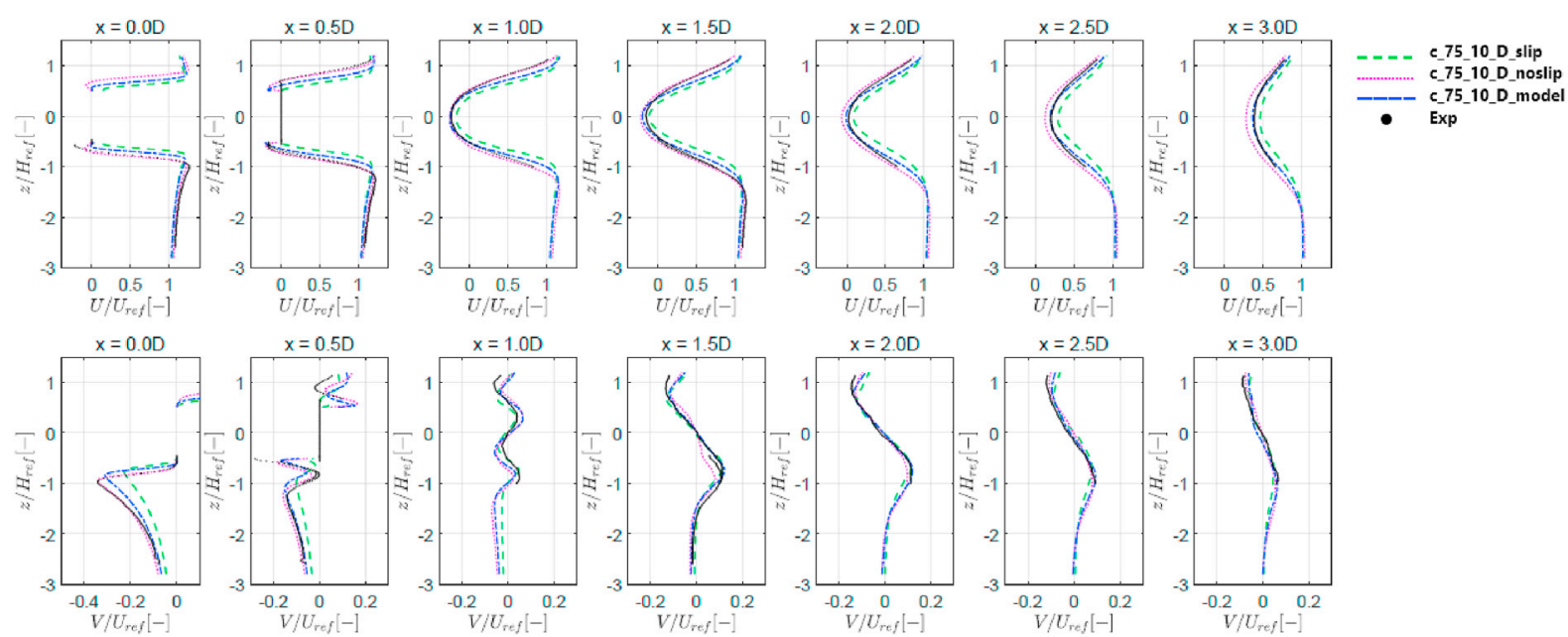

Fig. C.32. Streamwise (U) and cross-wind (V) mean velocity in the wake at $z=H_{r e f}$, using different boundary conditions at the building surface, slip condition (c_75_10_D_slip), no-slip condition (c_75_10_D_noslip) and turbulent wall model (c_75_10_D_model). 

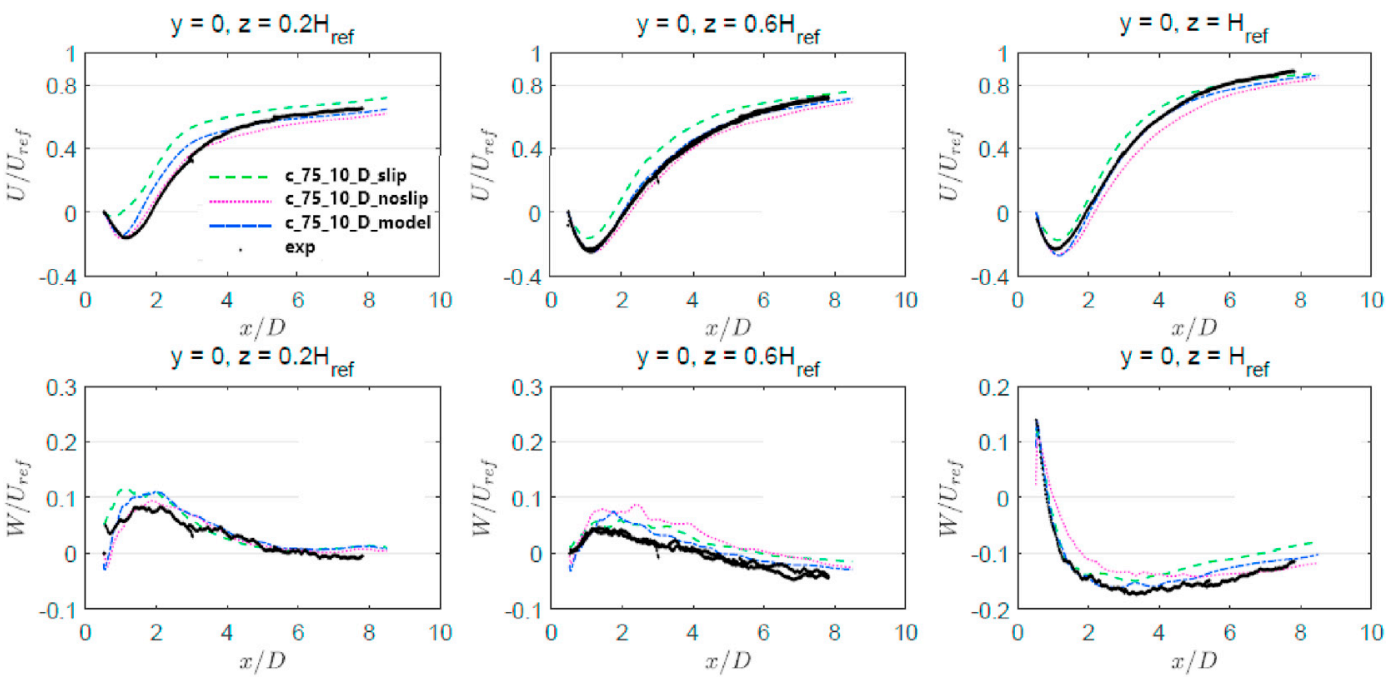

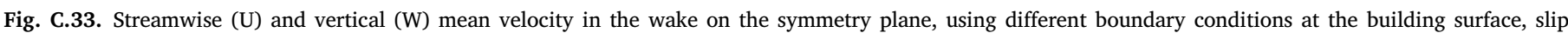
condition (c_75_10_D_slip), no-slip condition (c_75_10_D_noslip) and turbulent wall model (c_75_10_D_model).

The last point addressed here is the influence of the grid resolution at the building surface, which is illustrated in Fig. C.34. The finest grid (case f_75_10_slip) yields a better agreement with experimental data, especially in recirculation bubbles on the sides walls of the building. This is expected, since shear layers are very thin on these sides and that their spreading rate is a key parameter in the present flow dynamics. On the contrary, velocity profiles are very similar in the wake of the building, whose dynamics is governed by larger scales that are capture using both grids.
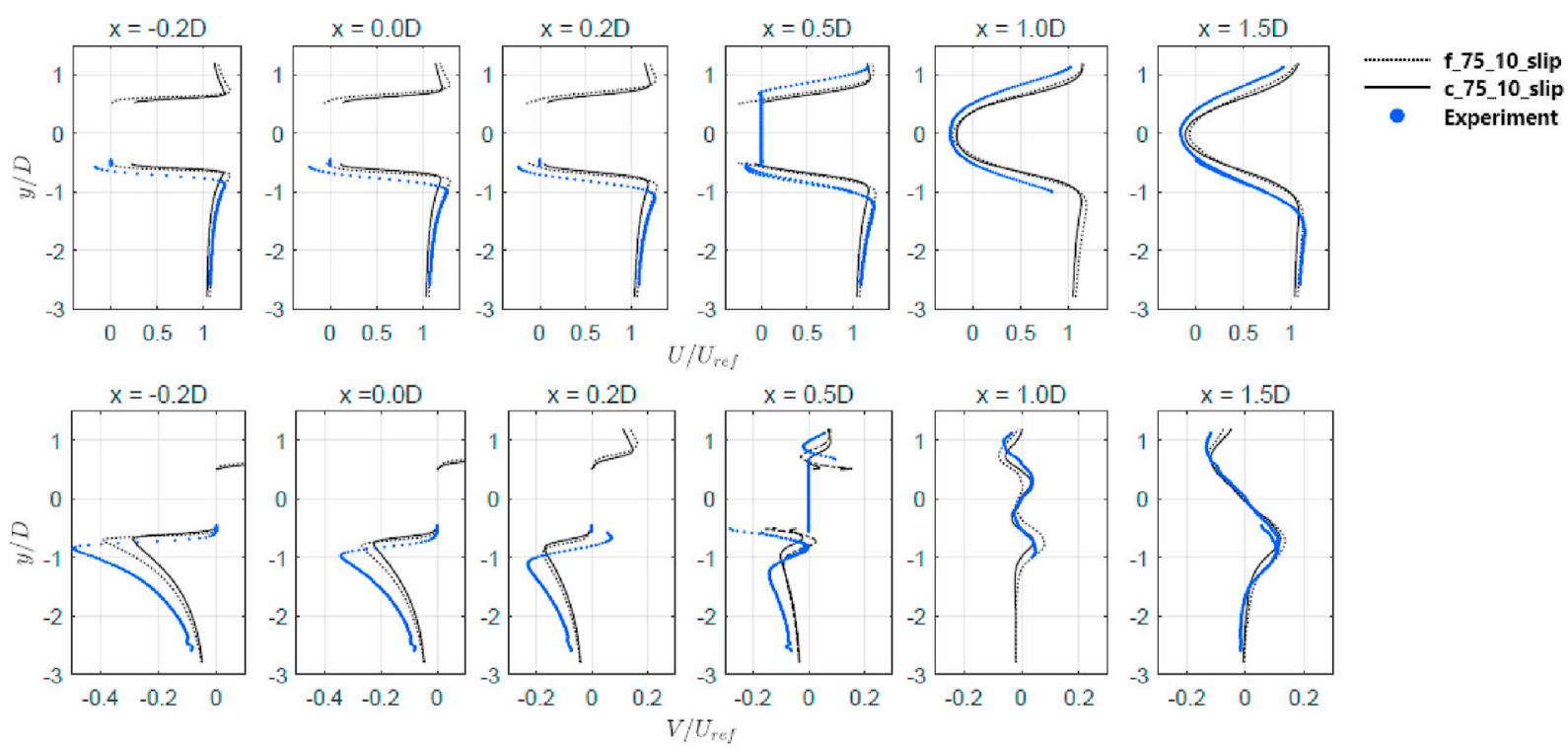

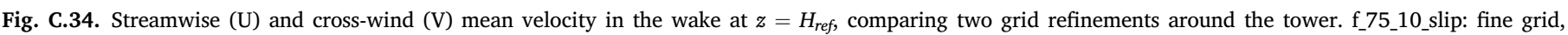
c_75_10_slip: coarse grid.

\section{References}

Aboshosha, H., Elshaer, A., Bitsuamlak, G., El Damatty, A., 2015. Consistent inflow turbulence generator for les evaluation of wind-induced responses for tall buildings. J. Wind Eng. Ind. Aerod. 142, 198-216.

Alminhana, G., Braun, A., Loredo-Souza, A., 2018. A numerical-experimental investigation on the aerodynamic performance of caarc building models with geometric modifications. J. Wind Eng. Ind. Aerod. 180, 34-48.
An, K., Fung, J., Yim, S., 2013. Sensitivity of inflow boundary conditions on downstream wind and turbulence profiles through building obstacles using a cfd approach. J. Wind Eng. Ind. Aerod. 115, 137-149.

Aristodemou, E., Boganegra, L., Mottet, L., Pavlidis, D., Constantinou, A., Pain, C. Robins, A., Apsimon, H., 2018. How tall buildings affect turbulent air flows and dispersion of pollution within a neighbourhood. Environ. Pollut. 233, 782-796. Bearman, P., Morel, T., 1983. Effect of free stream turbulence on the flow around bluff bodies. Prog. Aero. Sci. 20, 97-123. 
Blackburn, H., Melbourne, W., 1996. The effect of free stream turbulence on sectional lift forces on a circular cylinder. J. Fluid Mech. 306, 267-292.

Blocken, B., 2014. 50 years of computational wind engineering: past, present and future. J. Wind Eng. Ind. Aerod. 129, 69-102.

Blocken, B., 2015. Computational fluid dynamics for urban physics: importance, scales, possibilities, limitations and ten tips and tricks towards accurate and reliable simulations. Build. Environ. 91, 219-245. https://doi.org/10.1016/ j.buildenv.2015.02.015. fifty Year Anniversary for Building and Environment.

Blocken, B., Stathopoulos, T., van Beeck, J., 2016. Pedestrian-level wind conditions around buildings: review of wind-tunnel and cfd techniques and their accuracy for wind comfort assessment. Build. Environ. 100, 50-81. https://doi.org/10.1016/ j.buildenv.2016.02.004.

Bruno, L., Fransos, D., Coste, N., Bosco, A., 2010. 3D flow around a rectangular cylinder: computational study. J. Wind Eng. Ind. Aerod. 98, 263-276.

Cao, Y., Tamura, T., Kawai, H., 2020. Spanwise resolution requirements for the simulation of highreynolds-number flows past a square cylinder. Comput. Fluids 196, 104320.

Castro, I., Dianat, M., 1983. Surface flow patterns on rectangular bodies in thick boundary layers. J. Wind Eng. Ind. Aerod. 11, 107-119.

Castro, I., Robins, A., 1977. The flow around a surface-mounted cube in uniform and turbulent streams. J. Fluid Mech. 79, 307-335.

Corke, T., Nagib, H., 1979. Wind loads on a building model in a family of surface layers. J. Ind. Aerod. 5, 159-177.

Creech, A., Früh, W., Maguire, A., 2015. Simulations of an offshore wind farm using largeeddy simulation and a torque-controlled actuator disk model. Surv. Geophys. 36, $427-481$.

Dagnew, A., Bitsuamalak, G., 2013. Computational evaluation of wind loads on buildings: a review. Wind Struct. 16, 629-660.

Dagnew, A., Bitsuamalak, G., 2014. Computational evaluation of wind loads on standard building using les. Wind Struct. 18, 567-598.

Daniels, S., Castro, I., Xie, Z., 2013. Peak loading and surface pressure fluctuations of a tall model building. J. Wind Eng. Ind. Aerod. 120, 19-28.

Deck, S., Weiss, P., Renard, N., 2018. A rapid and low noise switch from rans to wmles on curvilinear grids with compressible flow solvers. J. Comput. Phys. 363, 231-255.

Dhamankar, N., Blaisdell, G., Lyrintzis, A., 2018. Overview of turbulent inflow boundary conditions for large-eddy simulations. AIAA J. 56, 1317-1334.

Elshaer, A., Aboshosha, H., Bitsuamlak, G., El Damatty, A., Dagnew, A., 2016. Les evaluation of wind-induced responses for an isolated and a surrounded tall building. Eng. Struct. 115, 179-195.

Eurocode, 2005. Eurocode 1: Actions on Structures-Part 1-4: General Actions-Wind Actions. Standard. European Committee for Standardisation (CEN).

Feng, Y., Boivin, P., Jacob, J., Sagaut, P., 2019a. Hybrid recursive regularized lattice Boltzmann simulation of humid air with application to meteorological flows. Phys. Rev. E 100, 023304

Feng, Y., Boivin, P., Jacob, J., Sagaut, P., 2019b. Hybrid recursive regularized thermal lattice Boltzmann model for high subsonic compressible flows. J. Comput. Phys. 394, 82-99.

Feng, Y., Guo, S., Jacob, J., Sagaut, P., 2019c. Solid wall and open boundary conditions in hybrid recursive regularized lattice Boltzmann method for compressible flows. Phys. Fluids 31, 126103.

Franke, J., Hellsten, A., Schlunzen, H., Carissimo, B., 2007. Best Practice Guideline for the CFD Simulation of Flows in the Urban Environment. COST 732: Quality Assurance and Improvement of Microscale Meteorological Models.

Garnier, E., Adams, N., Sagaut, P., 2009. Large-eddy Simulation for Compressible Flows. Springer, Berlin.

Gea-Aguilera, F., Gill, J., Zhang, X., 2017. Synthetic turbulence methods for computational aeroacoustic simulations of leading edge noise. Comput. Fluids 157 240-252.

Gerrard, J., 1966. The mechanics of the formation region of vortices behind bluff bodies. J. Fluid Mech. 25 (part 2), 401-413.

Gousseau, P., Blocken, B., van Heijst, G., 2013. Quality assessment of large-eddy simulation of wind flow around a high-rise building: validation and solution verification. Comput. Fluids 79, 120-133.

Guichard, R., 2019a. Assessment of an improved random flow generation method to predict unsteady wind pressures on an isolated building using large-eddy simulation. J. Wind Eng. Ind. Aerod. 189, 304-313.

Guichard, R., 2019b. Numerical simulation of wind effects on super high-rise buildings considering wind veering with height based on cfd. J. Fluid Struct. 91, 102715.

Guo, S., Feng, Y., Jacob, J., Sagaut, P., 2020. An efficient lattice Boltzmann method for industrial aerodynamics flows, I: basic model on D3Q19 lattice. J. Comput. Phys. , to appear.

Guo, Z., Shu, C., 2013. The Lattice Boltzmann Method and its Applications in Engineering. World Scientific.

Haque, M., Katsuchi, H., Yamada, H., Nishio, M., 2014. Investigation of flow fields around rectangular cylinder under turbulent flow by les. Eng. Appl. Comput. Fluid Mech. 8, 396-406.

Hearst, R., Gomit, G., Ganapathisubramani, B., 2016. Effect of turbulence on the wake of a wall-mounted cube. J. Fluid Mech. 804, 513-530.

Hillier, R., Cherry, N., 1981. The effects of free stream turbulence on separation bubbles. J. Wind Eng. Ind. Aerod. 8, 49-58.

Huang, S., Li, Q., Wu, J., 2010. A general inflow turbulence generator for large eddy simulation. J. Wind Eng. Ind. Aerod. 98, 600-617.

Jacob, J., Malaspinas, O., Sagaut, P., 2018. A new hybrid recursive regularised Bhatnagar-Gross-Krook collision model for lattice Boltzmann method-based large eddy simulation. J. Turbul. 19, 1051-1076.
Jacob, J., Sagaut, P., 2018. Wind comfort assessment by means of large eddy simulation with lattice Boltzmann method in full scale city area. Build. Environ. 139, 110-124.

Jarrin, N., Benhamadouche, S., Laurence, D., Prosser, R., 2006. A synthetic-eddy-method for generating inflow conditions for large-eddy simulations. Int. J. Heat Fluid Flow 27, 585-593.

Kim, Y., Castro, I., Xie, Z., 2013. Divergence-free turbulence inflow conditions for largeeddy simulations with incompressible flow solvers. Comput. Fluids 84, 56-68.

Kondo, K., Murakami, S., Mochida, A., 1997. Generation of velocity fluctuations for inflow boundary condition of les. J. Wind Eng. Ind. Aerod. 67-68, 51-64.

Kraichnan, R., 1970. Diffusion by a random velocity field. Phys. Fluids 13, 22-31.

Krüger, T., Kusumaatmaja, H., Kuzmin, A., Shardt, O., Silva, G., Viggen, E., 2017. The Lattice Boltzmann Method. Principles and Practice. Springer.

Lamberti, G., Garcia-Sanchez, C., Sousa, J., Gorlé, C., 2018. Optimizing turbulent inflow conditions for large-eddy simulation of the atmospheric boundary layer. J. Wind Eng. Ind. Aerod. 177, 32-44.

Lim, H., Castro, I., Hoxey, R., 2007. Bluff bodies in deep turbulent boundary layers: Reynolds-number issues. J. Fluid Mech. 571, 97-118.

Lim, H., Thomas, T., Castro, I., 2009. Flow around a cube in a turbulent boundary layer: les and experiments. J. Wind Eng. Ind. Aerod. 97, 96-109.

Longo, R., Ferrarotti, M., Sánchez, C.G., Derudi, M., 2017. Advanced turbulence models and boundary conditions for flows around different configurations of groundmounted buildings. J. Wind Eng. Ind. Aerod. 167, 160-182.

Luo, Y., Liu, H., Huang, Q., Xue, H., Lin, K., 2017. A multiscale synthetic eddu method for generating inflow data for les. Comput. Fluids 156, 103-112.

Luo, Y., Liu, H., Xue, H., Lin, K., 2018. Large-eddy simulation of wind loads on a high-rise building based on the multiscale synthetic eddy method. Adv. Struct. Eng. Special Issue 1-10.

Malaspinas, O., Sagaut, P., 2014. Wall model for large-eddy simulation based on the lattice Boltzmann method. J. Comput. Phys. 275, 25-40.

Martinuzzi, R., Tropea, C., 1993. Flow around surface-mounted, prismatic obstacles placed in a fully developed channel flow. J. Fluid Eng. 115, 85-92.

Merlier, L., Jacob, J., Sagaut, P., 2019. Lattice-Boltzmann large-eddy simulation of pollutant dispersion in complex urban environment with dense gas effect: model evaluation and flow analysis. Build. Environ. 148, 634-652. https://doi.org/ 10.1016/j.buildenv.2018.11.009.

Munoz-Esparza, D., Kosovic, B., van Beeck, J., Mirocha, J., 2015. A stochastic perturbation method to generate inflow turbulence in large-eddy simulation models: application to neutrally stratified atmospheric boundary layers. Phys. Fluids 27, 035102.

Nozu, T., Tamura, T., Takeshi, K., Akira, K., 2015. Mesh-adaptive les for wind load estimation of a high-rise building. J. Wind Eng. Ind. Aerod. 144, 62-69.

Pamiès, M., Weiss, P., Garnier, E., Deck, S., Sagaut, P., 2009. Generation of synthetic turbulent inflow data for large eddy simulation of spatially evolving wall-bounded flows. Phys. Fluids 21, 045103.

Pavlidis, D., Gorman, G., Gomes, J., Pain, C., Apsimon, H., 2010. Synthetic-eddy method for urban atmospheric flow modelling. Bound. Layer Meteorol. 136, 285-299.

Ricci, M., Patruno, L., Kalkman, I., de Miranda, S., Blocken, B., 2018. Towards les as a design tool: wind loads assessment on a high-rise building. J. Wind Eng. Ind. Aerod. $180,1-18$

Rodi, W., Ferziger, J., Breuer, M., Pourquie, M., 1997. Status of large eddy simulation: results of a workshop. J. Fluid Eng. 119, 248-262.

Roidl, B., Meinke, M., Schröder, W., 2013. A reformulated synthetic turbulence generation method for zonal rans-les method and its application to zero-pressure gradient boundary layers. Int. J. Heat Fluid Flow 44, 28-40.

Saathoff, P., Melbourne, W., 1997. Effect of free-stream turbulence on surface pressure fluctuations in a separation bubble. J. Fluid Mech. 337, 1-24.

Sagaut, P., 2005. Large Eddy Simulation for Incompressible Flows: an Introduction, third ed. Springer, Berlin.

Sagaut, P., Deck, S., 2009. Large eddy simulation for aerodynamics: status and perspectives. Phil. Trans. R. Soc. A 367, 2849-2860.

Sakamoto, H., 1985. Aerodynamic forces acting on a rectangular prism placed vertically in a turbulent boundary layer. J. Wind Eng. Ind. Aerod. 18, 131-151.

Sakamoto, H., Arie, M., 1982. Flow around a cubic body immersed in a turbulent boundary layer. J. Wind Eng. Ind. Aerod. 9, 275-293.

Sakamoto, H., Arie, M., 1983. Vortex shedding from rectangular prism and a circular cylinder placed vertically in a turbulent boundary layer. J. Fluid Mech. 126, $147-165$.

Sheng, R., Perret, L., Calmet, I., Demouge, F., Guilhot, J., 2018. Wind tunnel study of wind effects on a high-rise building at a scale 1:300. J. Wind Eng. Ind. Aerod. 174, $391-403$.

Skillen, A., Revell, A., Craft, T., 2016. Accuracy and efficiency improvements in synthetic eddy methods. Int. J. Heat Fluid Flow 62, 386-394.

Smirnov, R., Shi, S., Celik, I., 2001. Random flow generation technique for large-edy simulations and particule-dynamics modeling. J. Fluid Eng. 123, 359-371.

Sumer, B., Fredsoe, J., 2006. Hydrodynamics Around Cylindrical Structures, revised edition. World Scientific.

Tamura, T., 2008. Towards practical use of les in wind engineering. J. Wind Eng. Ind. Aerod. 96, 1451-1471.

Tamura, T., Nozawa, K., Kondo, K., 2008. Aij guide for numerical prediction of wind loads on buildings. J. Wind Eng. Ind. Aerod. 96, 1974-1984.

Taniguchi, S., Sakamoto, H., Arie, M., 1981. Flow around circular cylinders of finite height placed in a turbulent boundary layer. Bull. JSME 24 (187), 37-44.

Thordal, M., Bennetsen, J., Koss, H., 2019. Review for practical application of cfd for the determination of wind load on high-rise buildings. J. Wind Eng. Ind. Aerod. 186, 155-168. 
Tominaga, Y., Mochida, A., Yoshie, R., Kataoka, H., Nozu, T., Yoshikawa, M., Shirasawa, T., 2008. Aij guidelines for practical applications of cfd to pedestrian wind environment around buildings. J. Wind Eng. Ind. Aerod. 96, 1749-1761.

Vasaturo, R., Kalkman, I., Blocken, B., van Wesemael, P., 2018. Large-eddy simulation of the neutral atmospheric boundary layer: performance evaluation of three inflow methods for terrains with different roughness. J. Wind Eng. Ind. Aerod. 173, 241-261.

Vreman, A., 2004. An eddy-viscosity subgrid-scale model for turbulent shear flow: algebraic theory and applications. Phys. Fluids 16, 3670-3681.

Wang, Y., Chen, X., 2020. Simulation of approaching boundary layer flow and wind loads on high-rise buildings by wall-modeled les. J. Wind Eng. Ind. Aerod. 207, 104410.

Wilhelm, S., Jacob, J., Sagaut, P., 2018. An explicit power-law-based wall model for lattice Boltzmann method-Reynolds-averaged numerical simulations of the flow around airfoils. Phys. Fluids 30, 065111.
Wilhelm, S., Jacob, J., Sagaut, P., 2020. A new explicit algebraic wall model for les of turbulent flows under adverse pressure gradient. Flow, Turbul. Combust. 1-35.

Woodward, H., Stettler, M., Pavlidis, D., Aristodemou, E., Apsilmon, H., Pain, C., 2019 A large eddy simulation of the dispersion of traffic emissions by moving vehicles at an intersection. Atmos. Environ. 215, 116891.

Wu, X., 2016. Inflow turbulence generation methods. Annu. Rev. Fluid Mech. 49, 23-49.

Yan, B., Li, Q., 2015. Inflow turbulence generation methods with large-eddy simulation for wind effects on tall buildings. Comput. Fluids 116, 158-175.

Yan, B., Li, Q., 2017. Detached-eddy and large-eddy simulations of wind effects on a highrise structure. Comput. Fluids 150, 74-83.

Yu, R., Bai, X., 2014. A fully divergence-free method for generation of inhomogeneous and anisotropic turbulence with large spatial variation. J. Comput. Phys. 256, 234-253. 